\title{
Cálculo do Risco de Falha no Controle de Cheias: \\ Uma Abordagem por Equações Diferenciais Estocásticas
}

\section{Silvana Aparecida Meira}

Orientador: Prof. Dr. Marinho Gomes de Andrade Filho

"Dissertação apresentada ao Instituto de Ciências Matemáticas e de Computação - USP Campus de São Carlos, como parte dos requisitos necessários para a obtenção do título de mestre em Ciências, na área de Ciências de Computação e Matemática Computacional".

São Carlos

1998 


\section{Agradecimentos}

A realização deste trabalho não seria possível sem a ajuda de Deus, o apoio de algumas pessoas e instituições que, de alguma forma, contribuíram para esta dissertação e às quais expresso meus sinceros agradecimentos.

Ao Prof. Dr. Marinho Gomes de Andrade Filho, pela orientação primorosa, pelo exemplo de profissional, pelo incentivo, dedicação e por tudo que me ensinou nesse período de Mestrado.

À Profa. Dra. Maria Creusa Brettas Salles e ao Prof. Dr. Adriano A. F. M. Carneiro pelos comentários e sugestões no meu exame de qualificação.

Aos Professores do ICMC por tudo que contribuíram para minha formação.

À todos os meus amigos e especialmente a Claudia, Gilberto, Roberto, Marcia e Josenildo pelo incentivo, compartilhamento e pela amizade.

Aos funcionários do ICMC, às meninas da biblioteca e da seção de PósGraduação, que sempre prestam seus serviços com muita atenção e eficiência.

À CAPES, pelo apoio financeiro.

À minha família pelo carinho, apoio e incentivo. 


\section{Sumário}

\section{Capítulo 1}

Apresentação do Problema

1.1. Introdução

\section{Capítulo 2}

Relação Entre a Carga e Resistência ………................................................................ 5

2.1. Considerando Descarga de Referência Constante ………………......................... 5

2.2. Considerando Descarga de Referência Variável ..................................................... 7

\section{Capítulo 3}

Modelo Estocástico para $X(t)$ e $Y(t)$........................................................................ 9

3.1. Modelando a Carga ….............................................................................. 9

3.2. Modelando a Resistência $X(t)$ com Descarga de Referência Constante ......................... 10

3.3. Modelando a Resistência $X(t)$ com Descarga de Referência Variável ........................11

\section{Capítulo 4}

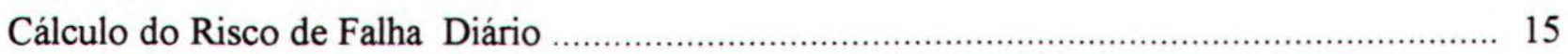

4.1. Descarga de Referência Constante ............................................................... 15

4.2. Descarga de Referência Variável …………................................................. 18

\section{Capítulo 5}

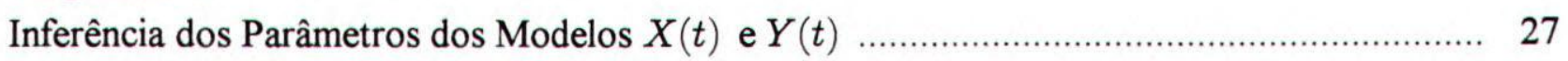

5.1. Estimadores de Máxima Verossimilhança com Descarga de Referência Constante ...... 27

5.2. Estimadores Bayesianos com Descarga de Referência Constante ............................. 30

5.2.1 Algoritmo Amostrador de Gibbs ............................................................. 33

5.3. Estimadores e Máxima Verossimilhança com Descarga de Referência Variável .......... 34

5.4. Estimadores Bayesianos com Descarga de Referência Variável ................................. 36 


\section{Capítulo 6}

Estudo de Caso

6.1. Comparação entre o Método Proposto e os Métodos existentes

\section{Capítulo 7}

Conclusão e Propostas Futuras

Referências Bibliográficas

Apêndice A

A. 1. Método da Curva Volume $X$ Duração ………................................................. 50

A.2. Método das Trajetórias Críticas .................................................................... 52

Apêndice B

B.1. Abordagem Bayesiana e Simulação de Monte Carlo em Cadeia de Markov ........... 55

B.2. O Amostrador de Gibbs ...................................................................... 57

B.3. O Critério de convergência de Gelman e Rubin .................................................. 59 


\section{Resumo}

Este trabalho propõe um modelo estocástico em conjunto com conceitos de análise de confiabilidade. Nestes modelos, a afluência que atinge o reservatório durante uma cheia é considerada como uma carga, e a capacidade do reservatório para amortecer esta cheia é considerada como uma resistência que o reservatório oferece à sua propagação. Aqui, a carga e a resistência são modeladas como processo do tipo difusão, e utiliza-se a fórmula de Itô para calcular o volume de espera associado a um determinado risco de falha. A inferência dos parâmetros do modelo é feita por meio de inferência bayesiana usando-se o algoritmo de simulação de Monte Carlo em cadeias de Markov. Os estimadores de máxima verossimilhança são usados para comparação. $\mathbf{O}$ método foi aplicado para um conjunto de dados utilizando-se nove anos de vazões afluentes diárias em período de cheia que chegam à usina de Xavantes. O volume de espera foi calculado pelo método proposto, usando-se descarga de referência constante, e comparado com os volumes obtidos por outros métodos existentes. $O$ método da curva volume $\times$ duração deixa um volume de espera constante durante todo o período chuvoso, usando-se somente $83,6 \%$ da capacidade do reservatório. $\mathrm{O}$ método proposto faz uma alocação dinâmica do volume de espera, utilizando-se $96.5 \%$ e $95.3 \%$ da capacidade do reservatório quando a abordagem bayesiana e o método de máxima verossimilhança são adotados, respectivamente. Dentre os métodos propostos, o método bayesiano mostrou-se mais vantajoso em relação ao desperdício de energia devido à utilização da capacidade do reservatório. $O$ método das trajetórias críticas necessita de séries com um comprimento significativo de vazões diárias, e na prática só pode ser aplicado com séries sintéticas de vazões. 


\section{Abstract}

This work proposes a stochastic model together with the concepts of reliability analysis. In these models, the inflow that reaches the reservoir during the flood is considered as a load, and the capacity of the reservoir for deaden this flood is considered as a resistance that the reservoir offers to its spreading. Here, the load and the resistance are modelled like a diffusion process, and it is used the Itô formula to calculate the waiting volume associate with a fail risk. The inference of the parameter of the model is done by Bayesian inference using the MCMC. The estimates of the maximum likelihood are used to compare. The method was applied together with the date record of mine year of daily streamflow during the period of the flood that come to Xavantes Power Station. The wait volume was calculated by the proposed method, using the discharge of the constant reference, and comparing with the volumes obtained through other existing methods. The curve volume $\times$ duration, leave a constant wait volume during the whole wet period, using onty $83.6 \%$ of the reservoir capacity. The method proposed does a dinamic allocation of the wait volume, using $96.5 \%$ and $95.3 \%$ of the capacity from the reservoir when the Bayesian approach and the maximum likelihood method are adopted, respectively. Within the proposed methods, the Bayesian method showed to be with more advantageons in relation to the waste of the energy due to the utilization of the reservoir capacity. The method of the critical trajectories need some series with the significant lenght of the daily discharge, in practive this only can applied with synthetic streamflow series. 


\section{Capítulo 1}

\section{Apresentação do Problema}

\subsection{Introdução}

O sistema brasileiro de geração de energia elétrica é um sistema hidrotérmico com forte predominância de geração hidroelétrica. Desta forma, a maioria dos reservatórios do sistema hidroelétrico foi projetada e construída visando exclusivamente a geração de energia elétrica. As estruturas dos vertedouros e os niveis máximos operativos dos reservatórios foram definidos, visando-se a segurança da barragem no caso de ocorrência de eventos de alta frequêencia, tais como cheias que só devem ocorrer a cada 1000 anos. Portanto, geralmente não é considerado, na fase de projeto e conseqüentemente na operação do sistema, o controle das enchentes que podem causar danos a jusante dos reservatórios. Um valioso levantamento histórico deste problema é apresentado em [Lopes et alii-1986]. O efeito de regularização propiciado pela criação dos reservatórios de aproveitamento hidroelétrico fez que as cheias de menor porte, ou de menor periodo de recorrência, ocorressem menos freqüentemente. Em conseqüência desse fato, áreas que eram periodicamente inundadas tornaram-se disponiveis e utilizáveis, embora sujeitas a um determinado risco de inundação. Com esta regularização, as populações e algumas instituições sentiram confiança em se aproximar das margens destes rios e passaram a utilizar estas áreas para diversos fins, tais como obras civis, agricultura e urbanização. Este fato deu origem a restriçб̃es para o escoamento dos rios, 
que vieram a se refletir na forma de operar estes reservatórios, acrescentando ao objetivo primário de geração de energia elétrica o objetivo de controle de cheias.

Para se efetuar o controle de cheias, é necessário prever a disponibilidade de volumes vazios nos reservatórios, capazes de absorver parcelas determinadas das afluências, evitando ou reduzindo o impacto de danos causados a jusante. No entanto, a manutenção de volumes de espera nos reservatórios conduz a uma redução da disponibilidade energética do aproveitamento e, por se tratar de um aproveitamento hidroelétrico, isto causa um aumento do risco de geração térmica futura. Torna-se evidente a existência de um conflito entre a utilização dos reservatórios para controle de cheias e para geração de energia elétrica. Para minimizar este conflito, é necessário, do ponto de vista energético, alocar o menor volume de espera possível. Mas, do ponto de vista do controle de cheias, é necessário se avaliar o risco de que o reservatório não seja capaz de controlar uma cheia quando um determinado volume de espera é alocado.

Entre os métodos utilizados para cálculo do volume de espera e risco de falha, um dos mais tradicionais é o Método da Curva Volume $\times$ Duração [Beard-1963]. A aplicação desse método apresenta algumas dificuldades, das quais as principais são as variações amostrais que fazem que a curva volume $\times$ duração não seja côncava, como é esperado, e a escolha de qual distribuição de probabilidade teórica deve ser ajustada aos dados. Uma outra dificuldade de aplicação desse método é que ele informa somente um único volume de espera para toda a estação chuvosa, não considerando a variação do potencial da cheia com o decorrer desta estação. Tal solução, na medida em que existem interesses conflitantes, não é eficiente.

Visando superar as dificuldades do Método da Curva Volume $\times$ Duração, em [Kelman1987] foi proposto um método denominado Método das Trajetórias Críticas. Este método permite determinar para cada dia da estação chuvosa, o volume de espera associado a um risco previamente estabelecido e garante, com certo risco, que as restrições de descarga não serão violadas sob a hipótese de que se repita qualquer situação hidrológica observada no passado. $\mathrm{O}$ método, contudo, 
não é próprio para as séries históricas usualmente disponíveis. Por exemplo, supondo-se um histórico de $n=30$ anos e um risco de $\rho=0.02$ (tempo de retorno de 50 anos), o número de trajetórias críticas não protegidas neste caso seria de $k=\rho n=0.6<1$. Portanto, a utilização desta metodologia só se viabiliza com a utilização de séries de comprimento significativo, o que só é obtido por meio da geração de séries sintéticas de vazões médias diárias.

Esse trabalho propõe um modelo estocástico em conjunto com conceitos de análise de confiabilidade. Uma revisão deste tipo de abordagem é feita em [Yen-1988] e mais recentemente este tipo de abordagem foi considerado em [Barbosa-1992] e [Meon-1992]. Nesses modelos, a afluência que atinge o reservatório durante uma cheia é considerada como uma carga, e a capacidade do reservatório para amortecer essa cheia é considerada como uma resistência que o reservatório oferece à sua propagação. Nos trabalhos de Yen e Meon, a carga e a resistência são consideras variáveis aleatórias independentes e invariantes no tempo. Isto simplifica o problema, mas é inadequado pois a carga e a resistência estão relacionadas pela equação dinâmica do reservatório como será mostrado neste trabalho. Aqui, a carga e a resistência são modelados como um processo estocástico do tipo difusão. Supondo-se que os processos são homogêneos para pequenos intevalos de tempo dentro do período chuvoso e utilizando-se a fórmula de Itô [Arnold-1974], formulou-se o problema de valor de contorno de Dirichlet cuja soluçăo numérica é uma aproximação para o risco de falha associado a uma carga e uma resistência . O método proposto permite que se façam considerações sobre a variação do potencial da cheia com o decorrer da estação chuvosa, permitindo-se que se calculem os volumes de espera associado a um determinado risco para diferentes intervalos de tempo dentro do periodo chuvoso. Com esse método, é possível fazer uma alocação dinâmica do volume de espera sem incorrer nas diffculdades encontradas pelo Método das Trajetórias Críticas.

Esta dissertação contém os seguintes capítulos: 
O capítulo 2, que apresenta a relação entre a carga e a resistência do reservatório através da equação de continuidade para o problema de controle de cheias, considerando-se na primeira etapa a descarga de referência constante, e em seguida essa relação é obtida para descarga de referência variável.

O capítulo 3 mostra a modelagem estocástica para a carga e a resistência do reservatório onde será considerado o uso de descarga de referência constante e em seguida variável. $\mathrm{O}$ modelo para a resistência quando, se tem descarga de referência constante, é visto como um caso particular da ocorrência em que a descarga de referência é variável.

No capítulo 4, calcula-se o risco de falha diário do reservatório usando-se o resultado do Lema 1; para o caso em que a descarga de referência é constante esse resultado é estendido com o Lema 2 para descarga variável. A falha do reservatório ocorre quando uma onda da cheia (carga) for maior do que a resistência do reservatório.

Os parâmetros do modelo apresentados no capítulo 3 são estimados no capítulo 5, usandose o método de máxima verossimilhança e uma abordagem bayesiana em conjunto com algoritmos de simulação de Monte Carlo em Cadeias de Markov. Para isso, será utilizada uma discretização diária para cada uma das trajetórias, correspondente a cada ano de observação durante o período chuvoso.

O capítulo 6 tratará da aplicação do método proposto na usina de Xavantes. $O$ conjunto de dados consiste de 9 anos de vazões diárias que chegaram ao reservatório de Xavantes nos meses de novembro a abril, nos anos que vão de 1981 à 1989.

No capítulo 6, apresenta-se a aplicação do método proposto e os resultados são comparados com os obtidos por meio de alguns métodos já existentes (ver apêndice A) para o cálculo do volume de espera.

As conclusões obtidas neste trabalho são apresentadas no capítulo 7. 


\section{Capítulo 2}

\section{Relação entre Carga e Resistência}

\subsection{Considerando Descarga de Referência Constante}

A forma mais fácil de formular o problema de confiabilidade de um reservatório para controle de cheias é considerar o volume de água que chega ao reservatório, em vez das vazões. Neste caso, vamos nos referir ao volume de água que chega ao reservatório durante uma cheia como a onda de cheia afluente. Considere-se um reservatório projetado para operar com um volume máximo $S_{1}$, um volume de espera $S_{2}$ para controle de cheias e um volume associado à dimensão de borda livre $S_{3}$ para conter as ondas induzidas pelo vento. Como mostra a Figura la. A cota $S_{\max }=S_{1}+S_{2}$ é chamada de max-maximoro. Na Figura 1 , apresenta-se um reservatório operando em um instante de tempot qualquer. Neste instante, denota-se por $q(t)$ a vazão afluente ao reservatório, por $v(t)$ a vazão liberada pelo vertedouro (vertimento), por $u(t)$ a vazão liberada pelas turbinas de usina hidroelétrica acoplada a barragem (turbinagem), por $S_{f b}$ o volume correspondente à borda livre, e por $v(t)+u(t)$ a descarga total (defluência). Na Figura Ib, pode-se notar também a resistência do reservatório $X(t)$, quando este opera com um volume armazenado $S(t)$. 


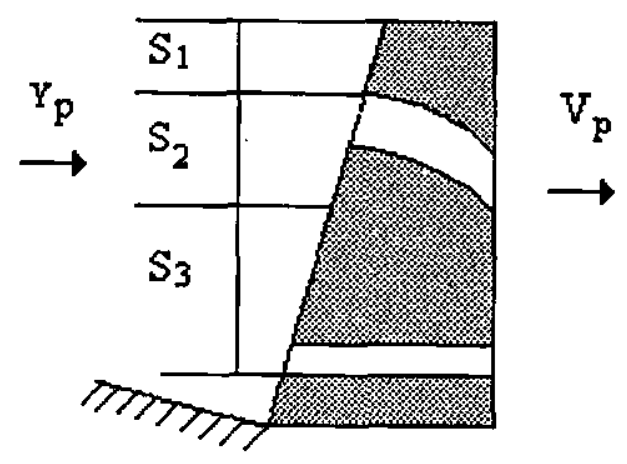

(a)

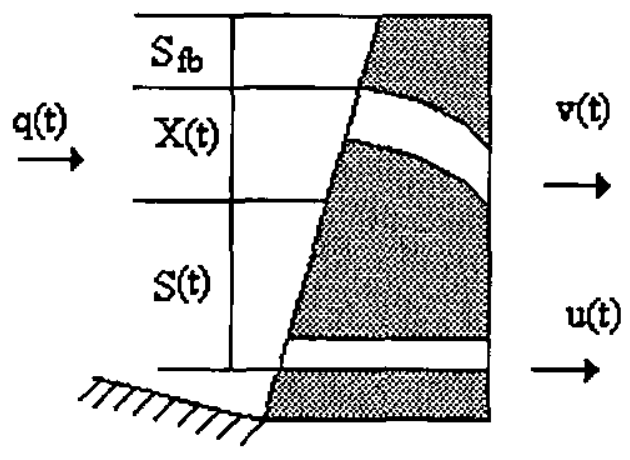

(b)

Figura 1(a) - Representação dos volumes de projeto.

1(b) - Representação dos volumes no instante $t$.

Considerando-se o reservatório operando com um volume armazenado $S(t)$ em um instante $t \in[0, T]$, então a resistência do reservatório é dada por:

$$
X(t)=S_{\max }-S(t)
$$

Considerando-se o intervalo de tempo $[t, t+\Delta t]$, a variação da resistência neste intervalo é dada por:

$$
X(t+\Delta t)-X(t)=-[S(t+\Delta t)-S(t)]
$$

A vazão defluente do reservatório no instante $t$ é dada por $v(t)+u(t)$, onde para controlar uma cheia, a máxima defluência que o reservatório pode liberar é a descarga de 
referência denotada pela vazão $q_{r}$. Assim, a equação de continuidade do reservatório no intervalo $[t, t+\Delta t]$ é dada por:

$$
S(t+\Delta t)=S(t)+\int_{t}^{t+\Delta t} q(s) d s-\int_{t}^{t+\Delta t} q_{r} d s
$$

A primeira integral em (2.3) representa a carga que atinge o reservatório no intervalo $[t, t+\Delta t]$. Essa integral pode ser escrita como:

$$
Y(t+\Delta t)=Y(t)+\int_{t}^{t+\Delta t} q(s) d s
$$

Desta forma, pode-se escrever a variação da resistência, substituindo-se (2.3) e (2.4) em (2.2); daí tem-se:

$$
\Delta X(t+\Delta t)=-\Delta Y(t+\Delta t)+q_{r} \Delta t
$$

A equação (2.5) mostra que a variação da resistência é igual à variação da carga com sinal trocado, acrescida do volume defluente neste intervalo, quando se adota como vazão defluente uma descarga de referência constante.

\subsection{Considerando Descarga de Referência Variável}

No caso em que a descarga de referência é variável, tem-se que a formulação do problema de confiabilidade do reservatório dá-se da mesma forma que no caso em que a descarga de referência é constante, modificando-se a equação (2.3), que passa a ser escrita como: 


$$
S(t+\Delta t)=S(t)+\int_{t}^{t+\Delta t} q(s) d s-\int_{t}^{t+\Delta t} r(s) d s
$$

Aqui $\{r(t), t \geq 0\}$ representa a descarga do reservatório no instante $t$ com $r(t)=u(t)+v(t)$. Desta forma, pode-se escrever:

$$
R(t+\Delta t)=R(t)+\int_{t}^{t+\Delta t} r(s) d s
$$

A equação para a variação da resistência é dada por (2.5) é modificada para:

$$
\Delta X(t+\Delta t)=-\Delta Y(t+\Delta t)+\Delta R(t)
$$

O próximo capitulo apresenta um modelo estocástico para as variáveis $X(t), Y(t)$ considerando ambos os casos descarga constante e descarga variável. 


\section{Capítulo 3}

\section{Modelo Estocástico para $X(t)$ e $Y(t)$}

A carga $Y(t)$ depende das condições meteorológicas, tais como chuva, temperatura, além das condições do solo e fluxos subterrâneos. Tudo isso atribui à carga um caráter estocástico. Propondose um modelo do tipo difusão para a carga, o modelo para a resistência é deduzido a partir da equação (2.5), que relaciona as variações entre carga e resistência.

\subsection{Modelando a Carga $Y(t)$}

Para modelar a carga, considera-se a vazão afluente $q(t)$, que chega ao reservatório em um instante do período de cheia, $t \in[0, T]$. Esta vazão pode ser modelada como um processo estocástico, que satisfaz o seguinte modelo:

$$
q(t)=\alpha(t)+\sigma(t) a(t)
$$

onde $\alpha(t)$ é uma função puramente determinista que representa a tendência e sazonalidade das vazões neste intervalo, e $a(t)$ é um processo independente e identicamente distribuído com distribuição normal $N(0,1)$. Esse ruído representa a flutuação aleatória das vazões em torno de $\alpha(t)$ 
com variância $\sigma^{2}(t)$. O processo $a(t)$ é chamado ruído branco e pode ser escrito como a derivada de um processo de Winner [Arnold-1973], $\{B(t), t \geq 0\}$. Assim, tem-se:

$$
a(t) \approx \lim _{\Delta t \rightarrow 0} \sigma(t) \frac{B(t+\Delta t)-B(t)}{\Delta t}=\sigma(t) \frac{d B(t)}{d t}
$$

Desta maneira, pode-se escrever a equação (3.1) de forma aproximada para qualquer $s \in[t, t+\Delta t] \subset[0, T]$, como:

$$
q(s) \approx \alpha(s)+\sigma(s) \frac{B(t+\Delta t)-B(t)}{\Delta t}
$$

A carga que atinge o reservatório no intervalo $[t, t+\Delta t]$ pode ser calculada substituindo-se (3.2) em (2.4), onde:

$$
Y(t+\Delta t)=Y(t)+\int_{t}^{t+\Delta t} \alpha(s) d s+\int_{t}^{t+\Delta t} \sigma(s)\left(\frac{B(t+\Delta t)-B(t)}{\Delta t}\right) d s
$$

Fazendo $\Delta t \rightarrow 0$, simbolicamente pode-se dizer que a carga $Y(t)$ satisfaz a seguinte equação diferencial estocástica:

$$
d Y(t)=\alpha(t) d t+\sigma(t) d B(t)
$$

\subsection{Modelando a Resistência $X(t)$ com Descarga de Referência Constante}

O modelo estocástico para a resistência do reservatório pode ser deduzido da equação (2.5), onde:

$$
\Delta X(t+\Delta t)=-\Delta Y(t+\Delta t)+q_{r} \Delta t
$$


Substituindo-se (3.3) em (3.5) e fazendo-se $\Delta t \rightarrow 0$, tem-se:

$$
d X(t)=\left(q_{r}-\alpha(t)\right) d t-\sigma(t) d B(t)
$$

NOTA 1: No problema de controle de cheias, as vazões são consideradas em intervalos de tempo diários; portanto, o período de cheia $[0, T]$ pode ser representado como a união de intervalos $I_{i}=\left[t_{i-1}, t_{i}\right]$. Quando $\Delta t=t_{i}-t_{i-1}=1$, cada intervalo $I_{i}$ representa um dia do período de cheia. Assim, $[0, T]=\cup_{i=1}^{N} I_{i}$, onde $N$ representa o número de dias da estação chuvosa. Aqui será considerado que os parâmetros $\alpha(t)$ e $\sigma(t)$ são constantes no intervalo $I_{i}$. Com essas consideraçōes, a equação diferencial estocástica para a resistência (equação 3.6) pode ser escrita como:

$$
d X(t)=\left(q_{r}-\alpha_{i}\right) d t-\sigma_{i} d B(t) \quad t \in I_{i}, i=1, \ldots, N
$$

Com a equação (3.7) é possivel calcular o risco de falha do reservatório para cada dia do intervalo $[0, T]$, como será mostrado no capítulo 4.

\subsection{Modelando a Resistência $X(t)$ com Descarga de Referência Variável}

$\mathrm{Na}$ equação (3.6), $q_{r}$ é a vazão defluente do reservatório, que tem sido considerada neste trabalho como uma constante igual à descarga de referência. No entanto, em se tratando de reservatórios de usinas hidroelétricas, essa descarga depende da política de operação do reservatório, que geralmente visa a minimização dos custos operacionais no atendimento de uma demanda de energia elétrica. Neste caso, o volume do reservatório $S(t)$ deve seguir uma trajetória ótima. Essa trajetória ótima é determinada de acordo com a meta de operação de longo e médio prazo (um a cinco anos). Desta forma, $S(t)$ é calculada defluindo-se do reservatório uma quantidade de água 
suficiente para que a trajetória $S(t)$ seja igual, ou pelo menos o mais próxima possivel dessa trajetória ótima. Porém, para se calcular a defluência necessária que fará a trajetória do reservatório $S(t)$ seguir a trajetória ótima é necessário que se saiba, ou pelo menos se estime, através de previsōes, a vazão afluente que chegará futuramente ao reservatório. Em uma situação real, a previsão da vazão afluente ao reservatório não pode ser feita sem algum grau de incerteza. Essas incertezas são chamadas erros de previsão. Neste caso, o modelo estocástico para $X(t)$, dado pela equação (3.6), pode ser modificado, reescrevendo-se a equação de continuidade do reservatório como:

$$
S(t+\Delta t)=S(t)+\int_{t}^{t+\Delta t} q(s) d s-\int_{t}^{t+\Delta t} r(s) d s,
$$

onde $r(t)$ é a vazão defluente que deve ser liberada pelo reservatório, levando-se em conta que $r(t)$ é calculada conhecendo-se somente uma previsão para $q(t)$. Então, adota-se um modelo estocástico para $r(t)$ que leva em conta o erro de previsão. Esse modelo é dado por:

$$
r(t)=\beta(t)+\gamma(t) b(t)
$$

onde $\beta(t)$ é uma função puramente determinista que representa a tendência e sazonalidade das vazōes neste intervalo e $b(t)$ é um ruído branco com distribuição normal $N(0,1)$. Esse ruído representa a flutuação aleatória das vazōes em torno de $\beta(t)$. O processo $b(t)$ pode ser escrito como a derivada de um processo de Winner [Arnold-1973], $\{W(t), t \geq 0\}$. Assim, tem-se:

$$
b(t) \approx \lim _{\Delta t \rightarrow 0} \gamma(t) \frac{W(t+\Delta t)-W(t)}{\Delta t}=\gamma(t) \frac{d W(t)}{d t}
$$

A equação (3.9) é escrita de forma aproximada para qualquer $s \in[t, t+\Delta t] \subset[0, T]$, como:

$$
r(s) \approx \beta(s)+\gamma(s) \frac{W(t+\Delta t)-W(t)}{\Delta t}
$$


$O$ volume de água que sai do reservatório no intervalo $[t, t+\Delta t]$ é dado por:

$$
R(t+\Delta t)=R(t)+\int_{t}^{t+\Delta t} \beta(s) d s+\int_{t}^{t+\Delta t} \gamma(s)\left(\frac{W(t+\Delta t)-W(t)}{\Delta t}\right) d s
$$

Fazendo-se $\Delta t \rightarrow 0$, simbolicamente pode-se dizer que $R(t)$ satisfaz a seguinte equação diferencial estocástica:

$$
d R(t)=\beta(t) d t+\gamma(t) d W(t)
$$

Substituindo-se (3.11) na equação de continuidade (3.8) e fazendo-se $\Delta t \rightarrow 0$, tem-se:

$$
d S(t)=d Y(t)-d R(t)
$$

Considerando-se agora a relação entre a resistência e o volume de água no reservatório, dada pela equação (2.2), tem-se:

$$
d X(t)=-d Y(t)+d R(t)
$$

Substituindo-se (3.4) e (3.12) em (3.14), obtém-se o modelo estocástico para a resistência, dado por:

$$
d X(t)=(\beta(t)-\alpha(t)) d t+\gamma(t) d W(t)-\sigma(t) d B(t)
$$

As equaçōes (3.4) e (3.15) modelam a carga e a resistência como um processo de Markov bidimensional, satisfazendo a equação diferencial estocástica: 


$$
\left(\begin{array}{l}
d X(t) \\
d Y(t)
\end{array}\right)=\left(\begin{array}{c}
\beta(t)-\alpha(t) \\
\alpha(t)
\end{array}\right) d t+\left(\begin{array}{cc}
\gamma(t) & -\sigma(t) \\
0 & \sigma(t)
\end{array}\right)\left(\begin{array}{c}
d W(t) \\
d B(t)
\end{array}\right)
$$

Considerando-se que os parâmetros $\alpha(t), \sigma(t)$ e $\gamma(t)$ são constantes no intervalo $I_{i}$, utilizando-se a Nota 1 , a equação diferencial estocástica bidimensional (equação 3.16) pode ser escrita como:

$$
\left(\begin{array}{c}
d X(t) \\
d Y(t)
\end{array}\right)=\left(\begin{array}{c}
\beta_{i}-\alpha_{i} \\
\alpha_{i}
\end{array}\right) d t+\left(\begin{array}{cc}
\gamma_{i} & -\sigma_{i} \\
0 & \sigma_{i}
\end{array}\right)\left(\begin{array}{c}
d W(t) \\
d B(t)
\end{array}\right)
$$

No capítulo posterior, dar-se-á o cálculo do risco de falha diário do reservatório, considerando-se a descarga de referência constante e variável. 


\section{Capítulo 4}

\section{Cálculo do Risco de Falha Diário}

\subsection{Descarga de Referência Constante}

Um reservatório falha no tocante ao controle de uma onda de cheia quando a sua defluência excede a descarga de referência. Para a situação que o reservatório está sempre operando com defluência constante e descarga igual a $q r$. Isso ocorre se a carga $Y(t)$ é maior que a resistência $X(t)$ em algum instante de tempo, obrigando o operador a defluir mais que o permitido. Assim, o risco de falha é definido como uma função do processo $\{Y(t), t \geq 0\}$, que representa a probabilidade de $Y(t)>X(t)$, para qualquer $t \in I_{i}$; ou seja, o risco de falha é definido como:

$$
\rho(X(t), Y(t))=P[Y(t)>X(t)] \quad t \in I_{i}
$$

Para avaliar o risco de falha segundo a equação (4.1), considera-se que a resistência $X(t)$ é limitada pela capacidade do reservatório: $X(t) \in R_{x}=\left[0, X_{\max }\right]$, em que $x_{t}$ denota um ponto nesse intervalo. E também, supõe-se que a carga está restrita a um conjunto, $Y(t) \in R_{y}=\left[0, Y_{\max }\right]$, em que $y_{t}$ denota um ponto nesse intervalo.

Indique-se por $\bar{F}=\{(X(t), Y(t)): Y(t) \geq X(t)\}$ a região onde o sistema falha $\mathrm{e}$ por $\bar{E}=\{(X(t), Y(t)): Y(t)<X(t)\}$ a região onde o sistema não falha . Considerando-se o processo 
$Y(t)$ e denotando-se a densidade de probabilidade conjunta de $Y(t)$ por $f_{Y(t)}\left(y_{t}\right)$ para $y_{t} \in \bar{E}$, podese calcular o risco de falha, dado por (4.1), como:

$$
P[Y(t)>X(t)]=\int_{x_{t}}^{\infty} f_{Y(t)}\left(y_{t}\right) d y_{t}
$$

Denota-se por:

$$
\rho\left(x_{t}\right)=\int_{x_{t}}^{\infty} f_{Y(t)}\left(y_{t}\right) d y_{t}
$$

O risco de falha do reservatório pode ser calculado como a probabilidade do processo que descreve a resistência do reservatório atingir a fronteira da região de falha $\partial F$, ou seja quando $X(t)=Y(t)$. Assim o risco de falha pode ser calculado como uma função da resistência, dada por:

$$
P[Y(t)>X(t)]=P[X(t) \in \partial F \mid X(t) \in \bar{E})
$$

ou seja:

$$
\rho\left(x_{t}\right)=\int_{X_{t} \in \partial F}^{\infty} f_{X(t)}\left(x_{t}\right) d x_{t}
$$

com $X(t)$ satisfazendo a equação diferencial estocástica $d X(t)=\left(q_{r}-\alpha_{i}\right) d t-\sigma_{i} d B(t)$.

Denotando-se por $\mathbb{C}^{0}\left(R_{x}\right)$ a classe de funções contínuas em $R_{x}$ e por $\mathbb{C}^{k}\left(R_{x}\right)$ a classe de funções que são contínuas e que possuem derivadas parciais contínuas em $R_{x}$, até a ordem $k$, o risco de falha é definido como uma função $\rho\left(x_{t}\right) \in \mathbb{C}^{2}(E) \cap \mathbb{C}^{0}\left(F \times R_{y}\right)$. Pode-se calcular o risco de falha com o resultado do seguinte Lema: 
Lema 1: Seja $\left\{X(t), t \in I_{i}\right\}$ o processo de Markov no espaço de probabilidade $(\bar{E}, P)$, satisfazendo a equação diferencial $d X(t)=\left(q_{r}-\alpha_{i}\right) d t-\sigma_{i} d B(t)$, e seja risco de falha $\rho\left(x_{t}\right) \in \mathbb{C}^{2}(E) \cap \mathbb{C}^{0}\left(F \times R_{y}\right)$, dado por:

$$
\rho\left(x_{t}\right)= \begin{cases}\exp \left\{-\frac{2\left(q_{r}-\alpha_{i}\right)}{\sigma_{i}^{2}}\left(x_{t}-y_{t}\right)\right\} & y_{t}<x_{t} \leq X_{\max } \\ 1 & 0 \leq x_{t} \leq y_{t}\end{cases}
$$

Prova: Seja $X(t)$ o processo que satisfaz a equação diferencial (3.6), e seja:

$$
\rho\left(x_{t}\right)=E_{X(t)}\left\{\rho\left(x_{t+\Delta t}\right)\right\} \quad(0 \leq t \leq t+\Delta t)
$$

onde $E_{X(t)}\{\cdot\}$ representa o valor esperado com relação a $f_{X(t)}\left(x_{t}\right)$. Então, escrevendo-se a förmula de Itô [Arnold-1974], tem-se:

$$
\begin{aligned}
\lim _{\Delta t \rightarrow 0} \frac{1}{\Delta t} E_{X(t)}\left\{\rho\left(x_{t+\Delta t}\right)-\rho\left(x_{t}\right)\right\}= \\
\quad=\lim _{\Delta t \rightarrow 0} \frac{1}{\Delta t} E_{X(t)}\left\{\int_{t}^{t+\Delta t} \mathcal{L} \rho\left(x_{s}\right) d \mathrm{~s}++\int_{t}^{t+\Delta t}\left(\nabla \rho\left(x_{s}\right)\right)^{\mathrm{T}} \cdot \sigma_{i} d B(s)\right\}
\end{aligned}
$$

onde: $\nabla$ é o vetor gradiente e $\mathcal{L}$ é o operador diferencial dado por:

$$
\mathcal{L}=\frac{\partial^{2}}{\partial x_{t}^{2}}+\frac{2\left(q_{r}-\alpha_{i}\right)}{\sigma_{i}^{2}} \frac{\partial}{\partial x_{t}}
$$

Sendo o valor esperado da integral estocástica igual a zero, logo:

$$
\lim _{\Delta t \rightarrow 0} \frac{1}{\Delta t} E_{X(t)}\left\{\rho\left(x_{t+\Delta t}\right)-\rho\left(x_{t}\right)\right\}=0
$$


Assim, para todo $x_{t} \in E$ tem-se que $\mathcal{L} \rho\left(x_{t}\right)=0$. Portanto, quando $x_{t}=y_{t}$ o risco satisfaz a condição de contorno $\rho\left(x_{t}\right)=1$. Então, a função $\rho\left(x_{t}\right)$ satisfaz o seguinte problema de valor de contorno:

$$
\text { Problema } \Sigma=\left\{\begin{array}{rc}
\mathcal{L} \rho\left(x_{t}\right)=0 & y_{t}<x_{t} \leq X_{\max } \\
\rho\left(x_{t}\right)=1 & 0 \leq x_{t} \leq y_{t}
\end{array}\right.
$$

cuja solução é a equação (4.5). Assim, conclui-se a prova do Lema.

\subsection{Descarga de Referência Variável}

Pode-se dizer que o reservatório falha no tocante ao controle de uma onda de cheia quando a defluência do reservatório excede o limite superior de defluência. Este limite pode ser a descarga de referência $q_{r}$, ou uma trajetória $u(t)$ dada por uma política ótima de operação. $\mathbf{O}$ desvio dessa trajetória ou a violação da descarga de referência ocorre se a carga $Y(t)$ é maior do que a resistência $X(t)$ em algum instante de tempo, obrigando o operador a defluir mais que o permitido. Assim, o risco de falha é definido como uma função do processo $(X(t), Y(t))$, que representa a probabilidade de $Y(t)>X(t)$, para qualquer $t \in[0, T]$; ou seja, o risco de falha é definido como:

$$
\rho(X(t), Y(t))=P[Y(t)>X(t)]
$$

Para avaliar o risco de falha segundo a equação (4.4), define-se o espaço de estado do processo $\{X(t), Y(t), t \geq 0\}$ como um subconjunto fechado $\bar{\Omega} \subset \mathbb{R}^{2}$ com fronteira $\partial \Omega$, em que $\left(x_{t}, y_{t}\right)$ denota um ponto em $\Omega$. Sejam agora $\bar{F}=\{(X(t), Y(t)): X(t) \leq Y(t)\}$ região de $\bar{\Omega}$ onde o

sistema falha e $\bar{E}=\{(X(t), Y(t)): X(t) \geq Y(t)\} \quad$ a região de $\bar{\Omega}$ onde o sistema não falha, e $\Gamma=\{(X(t), Y(t)): X(t)=Y(t)\}$ a região crítica onde a carga iguala-se a resistência. 
Considerando-se o processo $\Pi(t)=(X(t), Y(t))$ e denotando-se a densidade de probabilidade conjunta de $(X(t), Y(t))$ por $f_{\mathbf{I I}}\left(x_{t}, y_{t}\right)$ para $\left(x_{t}, y_{t}\right) \in \bar{E}$, pode-se calcular o risco de falha dado por (4.6) como:

$$
P[Y(t)>X(t)]=\int_{x_{t}}^{\infty} f_{Y(t)}\left(y_{t}\right) d y_{t}
$$

onde:

$$
f_{Y(t)}\left(y_{t}\right)=\int_{y_{t}}^{\infty} f_{\mathrm{II}}\left(x_{t}, y_{t}\right) d x_{t}
$$

Assim, tem-se:

$$
\rho\left(x_{t}, y_{t}, t\right)=\int_{x_{t}}^{\infty} \int_{y_{t}}^{\infty} f_{\text {II }}\left(x_{t}, y_{t}\right) d x_{t} d y_{t}
$$

Pode-se avaliar o risco de falha em intervalos de tempo $[t, t+\Delta t] \subset[0, T]$ pequeno em relação à duração do período úmido $T$, de forma que o processo $\{X(s), Y(s), t \leq s \leq t+\Delta t\}$ pode ser considerado estacionário nestes intervalos. Denotada-se por $\mathbb{C}^{0}(\Omega)$ (ou $\mathbb{C}^{0}(\partial \Omega$ )) a classe de funções contínuas em $\Omega$ (ou $\partial \Omega$ ), e por $\mathbb{C}^{k}(\Omega)$ a classe de funções que são contínuas e possuem derivadas parciais contínuas em $\Omega$, até a ordem $k$, o risco de falha é definido como uma função real $\rho\left(x_{t}, y_{t}\right): \bar{E} \mapsto[0,1]$, considerando-se $\rho\left(x_{t}, y_{t}\right) \in \mathbb{C}^{4}(E) \cap \mathbb{C}^{0}(\bar{E})$. Com as considerações feitas aqui, o cálculo do risco de falha passa a ser:

$$
\rho\left(x_{t}, y_{t}\right)=\int_{x_{t}}^{\infty} \int_{y_{t}}^{\infty} f_{\mathbf{I I}}\left(x_{t}, y_{t}\right) d x_{t} d y_{t} \forall\left(x_{t}, y_{t}\right) \in \bar{E}
$$

com $\Pi(t)=(X(t), Y(t))$ satisfazendo a equação diferencial estocástica:

$$
\Pi(t)=\boldsymbol{\theta}_{\boldsymbol{i}} d t+G_{\boldsymbol{i}} \boldsymbol{d} \boldsymbol{B}(t)
$$


onde se usou a seguinte notação:

$$
\theta_{i}=\left(\begin{array}{c}
\beta_{i}-\alpha_{i} \\
\alpha_{i}
\end{array}\right) \quad G_{i}=\left(\begin{array}{rr}
\gamma_{i} & -\sigma_{i} \\
0 & \sigma_{i}
\end{array}\right) \quad d B(t)=\left(\begin{array}{c}
d W(t) \\
d B(t)
\end{array}\right)
$$

Resolver a equação (4.10) analiticamente nem sempre é possível em problemas reais. Um resultado que permite avaliar o risco $\rho\left(x_{t}, y_{t}\right)$ sem ter que resolver a integral analiticamente é dado no seguinte Lema.

Lema 2: Sejam $\{\boldsymbol{\Pi}(t), t \geq 0\}$ o processo de Markov no espaço de probabilidade $(\mathbb{E}, P)$, satisfazendo a equação diferencial (4.11), e $\rho\left(x_{t}, y_{t}\right) \in \mathbb{C}^{4}(\Omega) \cap \mathbb{C}^{0}(\bar{E})$ o risco de falha dado pela equação (4.10). Então, a função $\rho\left(x_{t}, y_{t}\right)$ satisfaz o seguinte problema de valor de contorno:

$$
\text { Problema } \Sigma=\left\{\begin{aligned}
\mathcal{L} \rho\left(x_{t}, y_{t}\right)=0 & \left(x_{t}, y_{t}\right) \in E \\
\rho\left(x_{t}, y_{t}\right)=1 & \left(x_{t}, y_{t}\right) \in \Gamma \\
\rho\left(x_{t}, y_{t}\right)=0 & \left(x_{t}, y_{t}\right) \in \partial E \backslash \Gamma
\end{aligned}\right.
$$

onde $\Gamma$ é uma parte da fronteira $\partial E$ dada por $\Gamma=\{(X(t), Y(t)): X(t)=Y(t)\}$ e $\mathcal{L}$ é o operador diferencial definido por:

$$
\mathcal{L}=\frac{1}{2}\left(\gamma_{i}^{2}+\sigma_{i}^{2}\right) \frac{\partial^{2}}{\partial x_{t}^{2}}-\sigma_{i}^{2} \frac{\partial^{2}}{\partial x_{t} \partial y_{t}}+\frac{1}{2} \sigma_{i}^{2} \frac{\partial^{2}}{\partial y_{t}^{2}}+\left(\beta_{i}-\alpha_{i}\right) \frac{\partial}{\partial x_{t}}+\alpha_{i} \frac{\partial}{\partial y_{t}}
$$

Prova: Seja $\Pi(t)$ o processo que satisfaz a equação diferencial (4.10) e seja:

$$
\rho\left(x_{t}, y_{t}\right)=E_{\Pi(t)}\left\{\rho\left(x_{t+\Delta t}, y_{t+\Delta t}\right)\right\} \quad(0 \leq t \leq t+\Delta t)
$$


onde $E_{\Pi(t)}\{\cdot\}$ representa o valor esperado com relação a $f_{\Pi}\left(x_{t}, y_{t}\right)$. Então, escrevendo-se a fórmula de Itô [Arnold-1974], tem-se:

$$
\begin{aligned}
\lim _{\Delta t \rightarrow 0} \frac{1}{\Delta t} E_{\mathbf{\Pi}(t)}\left\{\rho\left(x_{t+\Delta t}, y_{t+\Delta t}\right)-\rho\left(x_{t}, y_{t}\right)\right\}= \\
\quad=\lim _{\Delta t \rightarrow 0} \frac{1}{\Delta t} E_{\mathbf{\Pi}(t)}\left\{\int_{t}^{t+\Delta t} \mathcal{L} \rho\left(x_{s}, y_{s}\right) d \mathbf{s}+\int_{t}^{t+\Delta t}\left(\nabla \rho\left(x_{s}, y_{s}\right)\right)^{\mathbf{T}} \cdot G_{i} d B(s)\right\}
\end{aligned}
$$

Sendo o valor esperado da integral estocástica igual a zero e $\rho\left(x_{t}, y_{t}\right) \in \mathbb{C}^{4}(\Omega) \cap \mathbb{C}^{0}(\bar{E})$, tem-se que:

$$
\lim _{\Delta t \rightarrow 0} \frac{1}{\Delta t} E_{\mathbf{\Pi}(t)}\left\{\rho\left(x_{t+\Delta t}, y_{t+\Delta t}\right)-\rho\left(x_{t}, y_{t}\right)\right\}=0
$$

Assim, para todo $\left(x_{t}, y_{t}\right) \in E$ em que $\mathcal{L} \rho\left(x_{t}, y_{t}\right)=0$, as condições de contorno são deduzidas imediatamente da definição do risco e da região $\Gamma$. Assim, conclui-se a prova do Lema.

NOTA 2: $O$ problema $\Sigma$ é conhecido como problema de valor de contorno de Dirichlet e pode ser resolvido numericamente por uma técnica, na qual tem-se mostrado mais precisa na solução desse tipo de problema, é a técnica apresentada em [Val e Andrade-1996]. Para isto, é necessário colocar o operador $\mathcal{L}$ na sua forma canônica. Isso pode ser feito através de transformações lineares adequadas [Garabedian-1964]. Para colocar o operador $\mathcal{L}$ dado em (4.12) na sua forma canônica, têm-se que reagrupar os termos e completar o quadrado. Logo:

$$
\mathcal{L}=\frac{1}{2} \gamma_{i}^{2} \frac{\partial^{2}}{\partial x_{t}^{2}}+\frac{1}{2} \sigma_{i}^{2}\left(\frac{\partial}{\partial x_{t}}-\frac{\partial}{\partial y_{t}}\right)^{2}+\left(\beta_{i}-\alpha_{i}\right) \frac{\partial}{\partial x_{t}}+\alpha_{i} \frac{\partial}{\partial y_{t}}
$$

Fazendo-se uma transformação de variáveis, tem-se: 


$$
\begin{array}{lr}
x_{t}=\gamma_{i} \xi_{t}+\sigma_{y} \eta_{t} & y_{t}=-\sigma_{i} \eta_{t} \\
\frac{\partial}{\partial \xi_{t}}=\gamma_{i}\left(\frac{\partial}{\partial x_{t}}\right) & \frac{\partial}{\partial \eta_{t}}=\sigma_{i}\left(\frac{\partial}{\partial x_{t}}-\frac{\partial}{\partial y_{t}}\right)
\end{array}
$$

Substituindo-se essa relação em (4.13), obtém-se o operador $\mathcal{L}$ na forma canônica dos operadores elípticos, ou seja:

$$
\mathcal{L}^{m}=\frac{1}{2}\left(\frac{\partial^{2}}{\partial \xi_{t}^{2}}+\frac{\partial^{2}}{\partial \xi_{t}^{2}}\right)+\frac{\beta_{i}}{\gamma_{i}} \frac{\partial}{\partial \xi_{t}}-\frac{\alpha_{i}}{\sigma_{i}} \frac{\partial}{\partial \eta_{t}}
$$

Então, a solução $\rho\left(x_{t}, y_{t}\right)$ do problema $\Sigma$ satisfaz a equação diferencial $\mathcal{L}^{*} \rho\left(\xi_{t}, \eta_{t}\right)=0$, ou seja:

$$
\frac{\partial^{2} \rho\left(\xi_{t}, \eta_{t}\right)}{\partial \xi_{t}^{2}}+\frac{\partial^{2} \rho\left(\xi_{t}, \eta_{t}\right)}{\partial \xi_{t}^{2}}+\frac{2 \beta_{i}}{\gamma_{i}} \frac{\partial \rho\left(\xi_{t}, \eta_{t}\right)}{\partial \xi_{t}}-\frac{2 \alpha_{i}}{\sigma_{i}} \frac{\partial \rho\left(\xi_{t}, \eta_{t}\right)}{\partial \eta_{t}}=0
$$

Denotando-se por $\Delta=\partial^{2} / \partial \xi_{t}^{2}+\partial^{2} / \partial \eta_{t}^{2}$ o operador laplaciano, pode-se escrever (4.16) como:

$$
\Delta \rho\left(\xi_{t}, \eta_{t}\right)+\frac{2 \beta_{i}}{\gamma_{i}} \frac{\partial \rho\left(\xi_{t}, \eta_{t}\right)}{\partial \xi_{t}}-\frac{2 \alpha_{i}}{\sigma_{i}} \frac{\partial \rho\left(\xi_{t}, \eta_{t}\right)}{\partial \eta_{t}}=0
$$

Uma simplificação em (4.17) pode ser obtida pela transformação:

$$
u\left(\xi_{t}, \eta_{t}\right)=\rho\left(\xi_{t}, \eta_{t}\right) \exp \left\{\frac{a_{1} \xi_{t}+a_{2_{i}} \eta_{t}}{2}\right\}
$$

onde

$$
a_{1_{i}}=\frac{2 \beta_{i}}{\gamma_{i}} \quad a_{2_{i}}=-\frac{2 \alpha_{i}}{\sigma_{i}}
$$


Definindo-se uma constante $\lambda_{i}^{2}=\frac{a_{1_{i}}^{2}+a_{2_{i}}^{2}}{2}$, pode-se escrever (4.17) como:

$$
\Delta u\left(\xi_{t}, \eta_{t}\right)-\lambda^{2} u\left(\xi_{t}, \eta_{t}\right)=0
$$

Através da transformação de variáveis (4.14) o problema $\Sigma$ pode ser transformado em um outro equivalente, que é indicado por Problema $\Sigma^{*}$, dado por:

$$
\text { Problema } \Sigma^{*}= \begin{cases}\Delta u\left(\xi_{t}, \eta_{t}\right)-\lambda^{2} u\left(\xi_{t}, \eta_{t}\right)=0 & \left(\xi_{t}, \eta_{t}\right) \in \mathcal{V} \\ u\left(\xi_{t}, \eta_{t}\right)=\exp \left\{\frac{a_{1_{i}} \xi_{t}+a_{2_{i}} \eta_{t}}{2}\right\} & \left(\xi_{t}, \eta_{t}\right) \in \Upsilon \\ \rho\left(\xi_{t}, \eta_{t}\right)=0 & \left(\xi_{t}, \eta_{t}\right) \in \partial \mathcal{V} \backslash \Upsilon\end{cases}
$$

Assumindo-se que as condições básicas para a existência de uma solução única do Problema $\Sigma^{*}$ são satisfeitas, e.g., [Garabedian-1964]; conhecendo-se a solução $u\left(\xi_{t}, \eta_{t}\right)$ do problema $\Sigma^{*}$, podese calcular a solução do problema $\Sigma$, pelas transformações inversas:

$$
\rho\left(x_{t}, y_{t}\right)=u\left(\xi_{t}\left(x_{t}, y_{t}\right), \eta_{t}\left(x_{t}, y_{t}\right)\right) \exp \left\{-\frac{a_{1_{i}} \xi_{t}\left(x_{t}, y_{t}\right)+a_{2_{i}} \eta_{t}\left(x_{t}, y_{t}\right)}{2}\right\}
$$

onde: $\quad a_{1_{i}}=\frac{2 \beta_{i}}{\gamma_{i}}$

$$
a_{2_{i}}=-\frac{2 \alpha_{i}}{\sigma_{i}}
$$

$$
\xi_{t}\left(x_{t}, y_{t}\right)=\frac{1}{\gamma_{i}}\left(x_{t}+y_{t}\right)
$$

$$
\eta_{t}\left(x_{t}, y_{t}\right)=-\frac{1}{\sigma_{i}} y_{t}
$$

Para resolver $\Sigma^{*}$ numericamente é necessário introduzir uma rede (grid) discreta em $(\mathcal{V} \cup \partial \mathcal{V})$ e avaliar $u\left(\xi_{t}, \eta_{t}\right)$, de forma aproximada em cada ponto (nó) da rede, adotando-se uma rede retangular uniforme ou rede quadrada, de acordo com a seguinte definição: 
Definição 1. Seja $S_{h}$ uma rede retangular uniforme ou rede quadrada com passo $h$ cobrindo a região retangular $\mathcal{V} \cup \partial \mathcal{V}$, tal que para qualquer $N$ e $M \quad \mathrm{~S}_{h}:=\left\{\left(\xi_{i}, \eta_{j}\right) \mid \xi_{i}=\xi_{0}+i h\right.$, $\eta_{j}=\eta_{0}+j h ; i=0,1,2, \cdots, N$ e $\left.j=0,1,2, \cdots, M\right\}$.

Um nó nesta rede é indicado por $\zeta_{i, j}=\left(\xi_{i}, \eta_{j}\right)$, e pode associar a toda função $\phi: \Omega \mapsto \mathbb{R}$ uma função discreta $\widehat{\phi}: S_{h} \mapsto \mathbb{R}$ chamada de função de rede, tal que $\widehat{\phi}_{i, j}=\phi\left(\zeta_{i, j}\right)$. Um nó $\zeta_{i, j}$ de $S_{h}$ é interno se a distância de $\zeta_{i, j}$ para $\partial \Omega$ é maior que $h$. O número total de pontos interiores é representado por $M_{0} N_{0}$, com $N_{0}=N-1$ e $M_{0}=M-1$.

Para uma função $u \in \mathbb{C}^{k}(\mathcal{V})$, seja $D_{k}$ o limite superior para a derivada de $k$-ésima ordem de $u$, isto é

$$
D_{k}:=\max \left\{\sup \left\{\left|\frac{\partial^{k} u\left(\xi_{t}, \eta_{t}\right)}{\partial \xi_{t}^{k-j} \eta_{t}^{j}}\right|:\left(\xi_{t}, \eta_{t}\right) \in \mathcal{V}\right\}: 0 \leq j \leq k\right\}
$$

Também, representa-se por $\mathrm{I}_{0}(\lambda h)$ a função de Bessel modificada de ordem zero, indicada pela série:

$$
\mathrm{I}_{0}(\lambda h)=\sum_{k=0}^{\infty} \frac{(\lambda h)^{2 k}}{2^{2 k}(k l)^{2}}
$$

Teorema 1. Sejam $u\left(\xi_{t}, \eta_{t}\right) \in \mathbb{C}^{4}(\Omega)$, a solução para o problema de valor de contorno $\Sigma ; S_{h}$ a rede quadrada, dada na definição 1 , e $u: S_{h} \mapsto \mathbb{R}$ a função de rede. Seja $U$ $\mapsto \mathbb{R}$ uma função de rede que satisfaz a seguinte equação de diferenças para $i=0,1,2, \cdots, N_{0}$, $j=0,1,2, \cdots, M_{0}$ :

$$
U_{i, j}=\frac{1}{4 \mathrm{I}_{0}(\lambda h)}\left(U_{i-1, j}+U_{i+1, j}+U_{i, j+1}+U_{i, j-1}\right)
$$


Segue que

$$
\|u-U\|_{\infty} \leq \frac{1}{\left(\mathrm{I}_{0}(\lambda h)-1\right)}\left[\frac{h^{4} D_{4}}{4 !}+\mathrm{O}\left(h^{6}\right)\right]
$$

Prova: [Val e Andrade, 1996]

Corolário 2. Seja $u\left(x_{t}, y_{t}\right)$ a solução do problema $\Sigma^{*}$ e $S_{h}$ a rede quadrada dada na Definição 1. Seja $U_{i, j}$ a função de rede, definida em $S_{h}$, que satisfaz a equação (4.20) para $i=1,2, \cdots N$ e $j=1,2, \cdots, M$. Então:

$$
\|u-U\|_{\infty}=\mathrm{O}\left(h^{2}\right)
$$

Prova: [Val e Andrade, 1996]

Usando-se as equações (4.19) e (4.20), pode-se calcular uma aproximação numérica para a solução do problema $\Sigma, \rho\left(x_{t}, y_{t}\right)$, em cada ponto da rede $\left(x_{i}, y_{j}\right)$, como segue:

$$
R_{i, j}=U_{i, j} \exp \left\{-\frac{a_{1} \xi_{i, j}+a_{2} \eta_{i, j}}{2}\right\}
$$

onde:

$$
\xi_{i, j}=\frac{1}{\gamma_{i}}\left(x_{i}+y_{j}\right) \quad \eta_{i, j}=-\frac{1}{\sigma_{i}} y_{j}
$$

Corolário 3. Seja $\rho\left(x_{t}, y_{t}\right)$ a solução do problema $\Sigma$ e seja $R_{i, j}$ a função de rede, definida em $S_{h}$, satisfazendo a equação (4.22) para $i=1,2, \cdots N$ e $j=1,2, \cdots, M$. Então: 


$$
\|\rho-R\|_{\infty}=\mathrm{O}\left(h^{2}\right)
$$

Prova: A prova é imediata das equações (4.23) e (4.24) e do Lema 2.

A inferência dos parâmetros dos modelos $X(t)$ e $Y(t)$ será feita no próximo capítulo, considerando-se a descarga de referência constante e variável. 


\section{Capítulo 5}

\section{Inferência dos Parâmetros dos Modelos $X(t)$ e $Y(t)$}

Nesse capítulo, considera-se o problema de estimação dos parâmetros dos modelos estocásticos. Quando se tem a descarga constante, os únicos parâmetros a serem estimados são os coeficientes de drift $\alpha_{\mathfrak{i}}$ e difusão $\sigma_{i}$, da carga $Y(t)$. No caso de descarga variável, os parâmetros a serem estimados são o vetor de drift $\theta_{i}$ e a matriz $(2 \times 2)$ de difusão $G_{i}$ da equação $(4.11)$. Serão apresentados o método de estimação de máxima verossimilhança e uma abordagem bayesiana; utilizando-se a simulação de Monte Carlo em cadeia de Markov para obter essas estimativas.

\subsection{Estimadores de Máxima Verossimilhança com Descarga de Referência Constante}

Para estimar os coeficientes da carga, considera-se uma versão discretizada do processo $Y(t)$ construída a partir do conjunto de observações do processo nos pontos $t_{i} \in I_{i}$ do conjunto $[0$, $T]=\cup{ }_{i=1}^{N} I_{i}$. Então, com probabilidade um, a trajetória de $Y(t)$ é completamente especificada quando a igualdade $Y_{i}=Y\left(t_{i}\right)$ é conhecida para todo $i$ [Basawa \& Rao-1980]. Considerando-se o intervalo de tempo $\Delta t=t_{i}-t_{i-1}=1$ dia, cada trajetória com $N$ dias corresponde a um período de cheia do ano, admitindo-se um só período de cheia por ano. Cada trajetória corresponde a um ano 
diferente. Portanto, o conjunto de dados consiste em $M$ trajetórias (anos) com $N$ observações em cada trajetória (dias) e denotando-se esses dados por $\left\{Y_{j, i}, i=0,1, \cdots, N\right.$ e $\left.j=1 \ldots M\right\}$. A densidade de probabilidade condicional para cada trajetória $j$ do processo $\left\{Y_{j, i}, i=0,1, \cdots, N\right\}$ é dada por:

$$
f_{j}\left(Y_{j, i} \mid Y_{j, i-1}, \alpha_{i}, \sigma_{i}^{2}\right)=\left(2 \pi \sigma_{i}\right)^{-1 / 2} \exp \left\{-\frac{1}{2 \sigma_{i}^{2}}\left(Y_{j, i}-Y_{j, i-1}-\alpha_{i}\right)^{2}\right\}
$$

Supondo-se $Y_{j, 0}$ conhecido, a função de verossimilhança para a trajetória $D_{j}=\left\{Y_{j, i}\right.$, $i=0,1, \cdots, N\}$, é dada por:

$$
L_{j}\left(\alpha_{i}, \sigma_{i}^{2} \mid D_{j}\right)=\prod_{i=1}^{N} f\left(Y_{j, i} \mid Y_{j, i-1}, \alpha_{i}, \sigma_{i}^{2}\right)
$$

Sendo $\Delta t=1$ dia, então $q_{j, i}=Y_{j, \mathrm{i}}-Y_{j, \mathrm{i}-1}$ é a vazão média diária que chega ao reservatório no dia $i$ do ano $j$. Indica-se por $q_{j}=\left(q_{j, 1}, \ldots, q_{j, N}\right)^{\prime}$ o vetor de vazões de um ano $j$, por $\boldsymbol{\alpha}=\left(\alpha_{1}, \ldots, \alpha_{N}\right)^{\prime}$ o vetor de drift e por $\boldsymbol{\Sigma}=\operatorname{diag}\left\{\sigma_{1}^{2}, \ldots \sigma_{N}^{2}\right\}$ a matriz de variância $N \times N$. Denotando-se por $|\Sigma|=\prod_{i=1}^{N} \sigma_{i}^{2}$, a função de verossimilhança (5.2) pode ser escrita como:

$$
L_{j}\left(\boldsymbol{\alpha}, \boldsymbol{\Sigma} \mid D_{j}\right) \propto|\boldsymbol{\Sigma}|^{-1 / 2} \exp \left\{-\frac{1}{2}\left(\boldsymbol{q}_{j}-\boldsymbol{\alpha}\right)^{\prime} \boldsymbol{\Sigma}^{-1}\left(\boldsymbol{q}_{j}-\boldsymbol{\alpha}\right)\right\}
$$

Considerando-se agora as $M$ trajetórias do processo $\left\{q_{j, i}, i=0,1, \ldots, N\right.$ e $\left.j=1 \ldots M\right\}$, então (5.3) representa a contribuição de uma trajetória para a função de verossimilhança completa que é dada por:

$$
L(\alpha, \boldsymbol{\Sigma} \mid D)=\prod_{j=1}^{M} L_{j}\left(\alpha, \boldsymbol{\Sigma} \mid D_{j}\right)
$$


onde $D=\left\{D_{j}, j=1 \ldots M\right\}$ denota o conjunto de todas $M$ trajetórias. Substituindo-se (5.3) em (5.4), tem-se:

$$
\begin{aligned}
& L(\boldsymbol{\alpha}, \boldsymbol{\Sigma} \mid D) \propto|\boldsymbol{\Sigma}|^{-M / 2} \prod_{j=1}^{M} \exp \left\{-\frac{1}{2}\left(\boldsymbol{q}_{j}-\boldsymbol{\alpha}\right)^{\prime} \boldsymbol{\Sigma}^{-1}\left(\boldsymbol{q}_{j}-\boldsymbol{\alpha}\right)\right\} \\
& L(\boldsymbol{\alpha}, \boldsymbol{\Sigma} \mid D) \propto|\boldsymbol{\Sigma}|^{-M / 2} \exp \left\{-\frac{1}{2} \sum_{j=1}^{M}\left(\boldsymbol{q}_{j}-\boldsymbol{\alpha}\right)^{\prime} \boldsymbol{\Sigma}^{-1}\left(\boldsymbol{q}_{j}-\boldsymbol{\alpha}\right)\right\}
\end{aligned}
$$

reescrevendo-se (5.6) tem-se:

$$
L(\boldsymbol{\alpha}, \boldsymbol{\Sigma} \mid D) \propto|\boldsymbol{\Sigma}|^{-M / 2} \exp \left\{-\frac{1}{2} \operatorname{tr}\left(\boldsymbol{\Sigma}^{-1} \boldsymbol{S}\right)\right.
$$

onde $S$ é uma matriz diagonal da soma dos quadrados relativo a $\alpha$, com os elementos da diagonal dados por:

$$
s_{i i}=\sum_{j=1}^{M}\left(q_{j, i}-\alpha_{i}\right)^{2}, \quad i=1,2, \ldots, N
$$

Para calcular os estimadores de máxima verossimilhança, pode-se escrever a equação (5.7) assim:

$$
L(\alpha, \boldsymbol{\Sigma} \mid \boldsymbol{D}) \propto|\boldsymbol{\Sigma}|^{-M / 2} \prod_{i=1}^{N} \exp \left\{-\frac{1}{2 \sigma_{i}^{2}} \sum_{j=1}^{M}\left(q_{j, i}-\alpha_{i}\right)^{2}\right\}
$$

Considerando-se a relação:

$$
\sum_{j=1}^{M}\left(q_{j, i}-\alpha_{i}\right)^{2}=(M-1) S_{i}^{2}+M\left(\alpha_{i}-\overline{q_{i}}\right)^{2}
$$


onde:

$$
\overline{q_{i}}=\frac{1}{M} \sum_{j=1}^{M} q_{j, i} \quad S_{i}^{2}=\frac{1}{(M-1)} \sum_{j=1}^{M}\left(q_{j, i}-\overline{q_{i}}\right)^{2}
$$

a função de verossimilhança (5.9) pode ser escrita na forma:

$$
L(\alpha, \Sigma \mid D) \propto|\Sigma|^{-M / 2} \prod_{i=1}^{N} \exp \left\{-\frac{1}{2 \sigma_{i}^{2}}\left[(M-1) S_{i}^{2}+M\left(\alpha_{i}-\overline{q_{i}}\right)^{2}\right]\right\}
$$

Tomando-se o logaritmo da equação (5.10) e derivando-se com relação a $\alpha_{i}$ e $\sigma_{i}^{2}$, tem-se os estimadores de máxima verossimilhança dados por:

$$
\widehat{\alpha}_{i}=\bar{q}_{i} \quad \text { e } \quad \widehat{\sigma}_{i}^{2}=\frac{M-1}{M} S_{i}^{2}, \quad i=1,2, \ldots, N
$$

As estimativas de $\alpha_{i}$ e $\sigma_{i}^{2}$ dadas por (5.11) podem ser substituídas em (4.5) para o cálculo do risco de falha. Ou fixando-se um risco de falha $p$, pode-se calcular o volume de espera para uma dada carga $y_{i}, i=1, \ldots, N$, usando-se a relação:

$$
x_{i}=y_{i}-\left(\frac{\widehat{\sigma}_{i}^{2}}{2\left(q_{r}-\widehat{\alpha}_{i}\right)}\right) \ln (p) \quad, i=1, \ldots, N
$$

\subsection{Estimadores Bayesianos com Descarga de Referência Constante}

No contexto bayesiano, os parâmetros $\alpha_{i}$ e $\sigma_{i}$ são considerados variáveis aleatórias. Assumindo-se densidades a priori para os parâmetros e combinando-se essas densidades com a função de verossimilhança através do teorema de Bayes, tem-se a densidade a posteriori para $\alpha_{i}$ e $\sigma_{i}$. Nesse contexto, os estimadores bayesianos são valores esperados de cada parâmetro com relação à sua densidade a posteriori. 
Para encontrar estimadores bayesianos para os parâmetros da equação (3.6), supõe-se que $\alpha_{i}$ e $\sigma_{i}$ são independentes e adota-se a densidade a priori não informativa de Jeffrey [Box e Tiao-1973]. Sob hipótese de a independência da densidade a priori para $\alpha$ ser constante e a densidade a priori conjunta será dada por:

$$
\pi_{0}(\boldsymbol{\alpha}, \boldsymbol{\Sigma})=\prod_{i=1}^{N}\left|-\frac{1}{N} \frac{\partial^{2} L(\boldsymbol{\alpha}, \boldsymbol{\Sigma} \mid \boldsymbol{D})}{\partial \sigma_{i}^{2}}\right|^{\frac{1}{2}}=|\boldsymbol{\Sigma}|^{-1}
$$

a densidade a posteriori é dada por, $\pi(\alpha, \Sigma \mid D) \propto L(\alpha, \Sigma \mid D) \pi_{0}(\alpha, \Sigma)$, que resulta em:

$$
\pi(\alpha, \Sigma \mid D) \propto|\Sigma|^{-\frac{\alpha+2}{2}} \exp \left\{-\frac{1}{2} \operatorname{tr}\left(\boldsymbol{\Sigma}^{-1} \boldsymbol{S}\right)\right\}
$$

Definindo-se o vetor $\bar{q}=\left(\bar{q}_{1}, \bar{q}_{2}, \ldots, \bar{q}_{N}\right)$, pode-se escrever a relação:

$$
\begin{aligned}
\exp \left\{-\frac{1}{2} \operatorname{tr}\left(\boldsymbol{\Sigma}^{-1} \boldsymbol{S}\right)\right\} & =\prod_{i=1}^{N} \exp \left\{-\frac{1}{2 \sigma_{i}^{2}}\left[(M-1) S_{i}^{2}+M\left(\alpha_{i}-\overline{q_{i}}\right)^{2}\right]\right\} \\
& =\exp \left\{-\frac{1}{2} \sum_{i=1}^{N} \frac{1}{\sigma_{i}^{2}}\left[(M-1) S_{i}^{2}+M\left(\alpha_{i}-\overline{q_{i}}\right)^{2}\right]\right\}
\end{aligned}
$$

onde:

$$
\exp \left\{-\frac{1}{2} \operatorname{tr}\left(\boldsymbol{\Sigma}^{-1} \boldsymbol{S}\right)\right\}=\exp \left\{-\frac{M}{2}(\boldsymbol{\alpha}-\overline{\boldsymbol{q}})^{\prime} \boldsymbol{\Sigma}^{-1}(\boldsymbol{\alpha}-\overline{\boldsymbol{q}})-\frac{1}{2} \sum_{i=1}^{N} \frac{1}{\sigma_{i}^{2}}\left[(M-1) S_{i}^{2}\right\}\right.
$$

Esta relação permite calcular a densidade condicional $\pi_{1}(\alpha \mid \Sigma, D)$, dada por:

$$
\pi_{1}(\alpha \mid \Sigma, D) \propto|\Sigma|^{-\frac{\alpha+2}{2}} \exp \left\{-\frac{1}{2}(\alpha-\bar{q})^{\prime} \Sigma^{-1}(\alpha-\bar{q})\right\}
$$




$$
\pi_{1}(\boldsymbol{\alpha} \mid \boldsymbol{\Sigma}, \boldsymbol{D}) \sim \operatorname{Normal}(\overline{\boldsymbol{q}}, \boldsymbol{\Sigma})
$$

A densidade condicional $\pi_{2}(\boldsymbol{\Sigma} \mid \alpha, D)$ é obtida diretamente da equação (5.14), e é dada por:

$$
\begin{aligned}
& \pi_{2}(\boldsymbol{\Sigma} \mid \boldsymbol{\alpha}, \boldsymbol{D}) \propto|\boldsymbol{\Sigma}|^{-\frac{M+2}{2}} \exp \left\{-\frac{1}{2} \operatorname{tr}\left(\boldsymbol{\Sigma}^{-1} \boldsymbol{S}\right)\right\} \\
& \pi_{2}(\boldsymbol{\Sigma} \mid \boldsymbol{\alpha}, \boldsymbol{D}) \sim \text { Wishart Inversa }((N-M-1) / 2, \boldsymbol{S} / 2)
\end{aligned}
$$

No contexto bayesiano, $\boldsymbol{\alpha}=\left(\alpha_{1}, \ldots, \alpha_{N}\right)^{\prime}$ e $\boldsymbol{\Sigma}=\operatorname{diag}\left\{\sigma_{1}^{2}, \ldots \sigma_{N}^{2}\right\}$ são variáveis aleatórias e portanto, para um risco fixo $p$, o volume de espera dado em (5.12) é visto como uma função desses parâmetros. Assim calcula-se o volume de espera associado ao risco $p$, tomando-se o valor esperado com relação a esses parâmetros, ou seja:

$$
x_{i}=y_{i}-E_{\pi(\alpha, \Sigma \mid D)}\left\{\frac{\sigma_{i}^{2}}{2\left(q_{r}-\alpha_{i}\right)}\right\} \ln (p) \quad, \quad i=1, \ldots, N
$$

Um método simples de avaliar o volume de espera dado em (5.17) é o de simulação de Monte Carlo em cadeia de Markov, usando-se o algoritmo amostrador de Gibbs [Casella-1994], para gerar uma amostra $\left\{\boldsymbol{\alpha}^{(k)}, \boldsymbol{\Sigma}^{(k)}, k=1,2, \ldots K\right\}$ da densidade a posteriori $\pi(\boldsymbol{\alpha}, \boldsymbol{\Sigma} \mid \boldsymbol{D})$. Com essa amostra, o estimador de Monte Carlo para o volume de espera é dado por:

$$
x_{i}=y_{i}-\frac{\ln (p)}{K} \sum_{k=1}^{K} \frac{\left(\sigma_{i}^{(k)}\right)^{2}}{2\left(q_{r}-\alpha_{i}^{(k)}\right)} \quad, \quad i=1, \ldots, N
$$




\subsubsection{Algoritmo Amostrador de Gibbs}

Uma rápida descrição do algoritmo amostrador de Gibbs é dada em seguida.

Para gerar uma amostra da densidade a posteriori $\pi(\alpha, \Sigma \mid D)$ usa-se o algoritmo amostrador de Gibbs, consideram-se as densidades condicionais:

$$
\begin{aligned}
& \pi_{1}(\boldsymbol{\alpha} \mid \boldsymbol{\Sigma}, \boldsymbol{D}) \sim N(\overline{\boldsymbol{q}}, \boldsymbol{\Sigma}) \\
& \pi_{2}(\boldsymbol{\Sigma} \mid \boldsymbol{\alpha}, \boldsymbol{D}) \sim I W[(N-M-1) / 2, \boldsymbol{S} / 2]
\end{aligned}
$$

O algoritmo amostrador de Gibbs consiste nos seguintes passos:

Passo 1: Inicializar o contador de iterações $k=1$ e arbitrar valores iniciais $\boldsymbol{\alpha}^{(k)}$ e $\boldsymbol{\Sigma}^{(k)}$.

Passo 2: Gerar novos valores das densidades condicionais (5.19) e (5.20), como segue:

$$
\begin{aligned}
& \boldsymbol{\alpha}^{(k+1)} \sim \pi_{1}\left(\boldsymbol{\alpha} \mid \boldsymbol{\Sigma}^{(k)}, \boldsymbol{D}\right) \\
& \boldsymbol{\Sigma}^{(k+1)} \sim \pi_{2}\left(\boldsymbol{\Sigma} \mid \boldsymbol{\alpha}^{(k)}, \boldsymbol{D}\right)
\end{aligned}
$$

Passo 3: Fazer $k \leftarrow k+1$ e voltar ao passo 2, até que $k=K$. 


\subsection{Estimadores de Máxima Verossimilhança com Descarga de Referência Variável}

Para encontrar os estimadores de máxima verossimilhança do vetor de drift e da matriz de difusão da equação (4.11), considera-se uma versão discretizada do processo bidimensional $\Pi(t)$ (definido no capitulo 4). A função de máxima verossimilhança é construída a partir de observaçōes desse processo nos pontos $t_{i} \in I_{i}$ do conjunto $[0, T]=\cup_{i=1}^{N} I_{i}$, considerando-se os intervalos de tempo $\Delta t=t_{i}-t_{i-1}=1$ dia. Cada trajetória com $N$-dias de observação corresponde a um dia de cheia de um determinado ano. Denotam-se as observações dessas trajetórias por $\Pi_{i, j}=\Pi_{j}\left(t_{i}\right)$, onde $j$ é o índice que indica o ano em que foi feita a observação do processo $\mathbf{I}(t)$. Assim, $\Pi_{i, j}$ significa as observações feitas no dia $i$ do ano $j$ e tem-se o conjunto total de observações indicado por $D=$ $\left\{\boldsymbol{\Pi}_{i, j}, i=, 1, \cdots, N ; j=1, \ldots, M\right\}$.

A densidade de probabilidade condicional do vetor $\left\{\Pi_{j, i}, i=, 1, \cdots, N ; j=1, \ldots, M\right\}$ é dada por:

$$
\begin{aligned}
f_{i}\left(\Pi_{j, i} \mid \Pi_{j, i-1}, \theta_{i}, M_{i}\right)=\left(2 \pi\left|\boldsymbol{M}_{i}\right|\right)^{-1 / 2} \\
\exp \left\{-\frac{1}{2}\left(\Pi_{j, i}-\Pi_{j, i-1}-\theta_{i}\right)^{\prime} \boldsymbol{M}_{i}^{-1}\left(\Pi_{j, i}-\Pi_{j, i-1}-\theta_{i}\right)\right\}
\end{aligned}
$$

Sendo $\boldsymbol{M}_{\boldsymbol{i}}=G_{i} G_{i}^{\prime},\left|\boldsymbol{M}_{\boldsymbol{i}}\right|$ indica o determinante da matriz $\boldsymbol{M}_{\boldsymbol{i}}$.

Considerando-se que $\circ$ processo $\left\{\boldsymbol{\Pi}_{j, i}, i=1, \cdots, N ; j=1, \ldots, M\right\}$ é um processo de Markov da densidade conjunta para a trajetória $D_{j}=\left\{\Pi_{j, i}, i=0,1, \cdots, N ; j=1, \ldots, M\right\}$, dada por:

$$
f_{i}\left(\Pi_{j, i}, \Pi_{j, i-1}, \cdots, \Pi_{1} \mid \boldsymbol{\theta}_{i}, \boldsymbol{M}_{\mathbf{i}}\right)=\prod_{i=1}^{N} f_{j}\left(\mathbf{\Pi}_{j, i} \mid \Pi_{j, i-1}, \boldsymbol{\theta}_{\boldsymbol{i}}, \boldsymbol{M}_{\boldsymbol{i}}\right)
$$


A função de verossimilhança de $\theta_{i}$ e $M_{i}$ é dada por:

$$
\begin{aligned}
& L_{j}\left(\theta_{i}, M_{i} \mid D_{j}\right)=\left(\prod_{i=1}^{N}\left(2 \pi M_{i}\right)^{-1 / 2}\right) \times \\
& \exp \left\{-\frac{1}{2} \sum_{i=1}^{N}\left(\Pi_{j, i}-\Pi_{j, i-1}-\theta_{i}\right)^{\prime} M_{i}^{-1}\left(\Pi_{j, i}-\Pi_{j, i-1}-\theta_{i}\right)\right\}
\end{aligned}
$$

Considerando as trajetórias de cada ano $j=1,2, \ldots, M$, temos:

$$
L\left(\theta_{i}, M_{i} \mid D_{N}\right)=\prod_{j=1}^{M} L_{j}\left(\theta_{i}, M_{i} \mid D_{j}\right)
$$

Então:

$$
\begin{aligned}
& L\left(\boldsymbol{\theta}_{i}, M_{i} \mid D_{j}\right)=\prod_{j=1}^{M}\left(\prod_{i=1}^{N}\left(2 \pi M_{i}\right)^{-1 / 2}\right) \exp \left\{-\frac{1}{2} \sum_{i=1}^{N}\left(\Pi_{j, i}-\Pi_{j, i-1}-\boldsymbol{\theta}_{i}\right)^{\prime} \boldsymbol{M}_{i}^{-1}\left(\mathbf{\Pi}_{j, i}-\Pi_{j, i-1}-\boldsymbol{\theta}_{i}\right)\right\} \\
& L\left(\boldsymbol{\theta}_{i}, \boldsymbol{M}_{\boldsymbol{i}} \mid D_{j}\right)=\left(\prod_{i=1}^{N}\left(2 \pi \boldsymbol{M}_{\mathbf{i}}\right)\right)^{-M / 2} \exp \left\{-\frac{1}{2} \sum_{j=1}^{M} \sum_{i=1}^{N}\left(\boldsymbol{\Pi}_{j, i}-\boldsymbol{\Pi}_{j, i-1}-\boldsymbol{\theta}_{i}\right)^{\prime} \boldsymbol{M}_{\boldsymbol{i}}^{-1}\left(\boldsymbol{\Pi}_{j, i}-\mathbf{\Pi}_{j, i-1}-\boldsymbol{\theta}_{\mathbf{i}}\right)\right\}
\end{aligned}
$$

Invertendo-se os somatórios, tem-se:

$$
L\left(\boldsymbol{\theta}_{i}, \boldsymbol{M}_{i} \mid D_{j}\right)=\left(\prod_{i=1}^{N}\left(2 \pi M_{i}\right)\right)^{-\mathrm{M} / 2} \exp \left\{-\frac{1}{2} \sum_{i=1}^{N} \sum_{j=1}^{M}\left(\Pi_{j, i}-\Pi_{j, i-1}-\boldsymbol{\theta}_{i}\right)^{\prime} \boldsymbol{M}_{i}^{-1}\left(\Pi_{j, i}-\Pi_{j, i-1}-\boldsymbol{\theta}_{i}\right)\right\}
$$

Tomando-se o $\log$ em (5.23) e fazendo-se $\log L\left(\theta_{i,} M_{i} \mid D_{j}\right)=l\left(\theta_{i,} M_{i} \mid D_{j}\right)$, tem-se:

$$
l\left(\boldsymbol{\theta}_{\boldsymbol{i}}, \boldsymbol{M}_{\boldsymbol{i}} \mid D_{j}\right) \propto\left(-\frac{M}{2}\right)\left(\sum_{i=1}^{N} \log \left(\boldsymbol{M}_{\boldsymbol{i}}\right)\right)-\frac{1}{2} \sum_{i=1}^{N}\left[\sum_{j=1}^{M}\left(\boldsymbol{\Pi}_{j, i}-\boldsymbol{\Pi}_{j, i-1}-\boldsymbol{\theta}_{\mathbf{i}}\right)^{\prime} \boldsymbol{M}_{\boldsymbol{i}}^{-1}\left(\boldsymbol{\Pi}_{j, i}-\mathbf{\Pi}_{j, i-1}-\boldsymbol{\theta}_{\boldsymbol{i}}\right)\right]
$$


Tomando-se as derivadas:

$$
\begin{aligned}
& \frac{\partial l\left(\theta_{i}, M_{i} \mid D_{j}\right)}{\partial \theta_{i}}=\sum_{j=1}^{M}\left(\Pi_{j, i}-\Pi_{j, i-1}-\theta_{i}\right)=0, \quad i=1,2, \ldots, N \\
& \frac{\partial l\left(\theta_{i}, M_{i} \mid D_{j}\right)}{\partial M_{i}}=-\left(\frac{M}{2}\right) M_{i}^{-1}-\frac{1}{2} S M_{i}^{-2}=0, i=1,2, \ldots ., N
\end{aligned}
$$

onde: $\boldsymbol{S}=\sum_{j=1}^{M}\left(\boldsymbol{\Pi}_{j, i}-\Pi_{j, i-1}-\theta_{i}\right)\left(\Pi_{j, i}-\Pi_{j, i-1}-\theta_{i}\right)^{\prime}$

os estimadores de máxima verossimilhança para $\theta_{i}$ e $M_{i}$ são dados por:

$$
\begin{aligned}
& \widehat{\boldsymbol{\theta}}_{i}=\frac{1}{\mathrm{M}} \sum_{\mathbf{i}=1}^{\mathrm{M}}\left(\Pi_{j, i}-\Pi_{j, i-1}\right) \\
& \widehat{M}_{i}^{-1}=\frac{1}{M} \sum_{i=1}^{M}\left(\dot{\Pi}_{j, i}-\Pi_{j, i-1}-\widehat{\boldsymbol{\theta}}_{i}\right)\left(\Pi_{j, i}-\Pi_{j, i-1}-\widehat{\boldsymbol{\theta}}_{i}\right)^{\prime} \\
& i=1,2, \ldots, N
\end{aligned}
$$

\subsection{Estimadores Bayesianos com Descarga de Referência Variável}

No contexto bayesiano, os parâmetros $\theta_{i}$ e $\boldsymbol{M}_{i}$ são considerados variáveis aleatórias. Assumindo-se densidades a priori para os parâmetros e combinando-se essas densidades com a função de verossimilhança através do teorema de Bayes, tem-se a densidade a posteriori para $\theta_{i}$ e $\boldsymbol{M}_{i}$. Nesse contexto, os estimadores bayesianos são valores esperados de cada parâmetro com relação à sua densidade a posteriori.

Adotando-se densidade a priori não informativa de Jeffrey [Box e Tiao-1973] e supondo-se que $\theta_{i}$ e $M_{i}$ são independentes, tem-se: 


$$
\pi_{0}\left(\theta_{i}, M_{i} \mid D\right) \propto M_{i}^{-1} ; \quad i=1,2, \ldots, N
$$

A densidade a posteriori é dada por:

$$
\pi\left(\theta_{i}, M_{i} \mid \cdot D\right) \propto L\left(\theta_{i}, M_{i} \mid D_{j}\right) \Pi_{0}\left(\theta_{i}, M_{i} \mid D\right) ; \quad i=1,2, \ldots, N
$$

As densidades condicionais podem ser usadas em um eventual algoritmo de MCMC para gerar $\theta_{i}$ e $\boldsymbol{M}_{\boldsymbol{i}}$.

Observa-se, neste caso, que as dificuldades aumentam para a aplicação dos algoritmos de MCMC, pois será necessário gerar amostras multidimensionais. No entanto, apesar dessa dificuldade adicional, o método bayesiano é perfeitamente aplicado para o caso.

No próximo capítulo, será realizado um estudo de caso para o reservatório de Xavantes usando descarga de referência constante. 


\section{Capítulo 6}

\section{Estudo de Caso}

O modelo de controle de cheia desenvolvido neste trabalho foi aplicado a um reservatório do sistema hidroelétrico do Sudeste Brasileiro, a Usina de Xavantes. Ela, pertencente à Companhia Energética de São Paulo - CESP, situada no Rio Paranapanema, afluente do Rio Paraná, na fronteira entre os estados de São Paulo e Paraná. Faz parte de um grande complexo de usinas hidraulicamente interligadas, composto por 35 usinas hidroelétricas, na que pode ser chamada de Grande Bacia do Rio Paraná, tendo a jusante de todas elas a Usina de Itaipu.

A Usina de Xavantes integra uma cascata de 6 usinas hidroelétricas, 3 a reservatório, entre as quais a própria Xavantes, e três a fio d’água, sendo a segunda de montante a jusante. A potência instalada é de $416 \mathrm{MW}$ e as restrições mínima e máxima de jusante (descarga de referência) são de $100 \mathrm{~m}^{3} / \mathrm{s}$ e $1800 \mathrm{~m}^{3} / \mathrm{s}$ respectivamente. O engolimento máximo (turbinagem) é de $648 \mathrm{~m}^{3} / \mathrm{s}$ e a capacidade total de vertimento é de $3252 \mathrm{~m}^{3} / \mathrm{s}$. O volume de espera projetado é de $S_{2}=615 \mathrm{~km}^{3}$. Os dados referentes às dimensø̃es do reservatório encontram-se descritos na Tabela 1 . 
Tabela 1: Dados do reservatório de Xavantes

\begin{tabular}{|l|c|c|}
\hline & Volume $\left(\mathrm{Km}^{3}\right)$ & Nível $(\mathrm{m})$ \\
\hline Volume útil & 3.041 & - \\
\hline Coroamento da barragem & - & 479.00 \\
\hline Max-maximoro & 9.410 & 475.50 \\
\hline Máximo operativo normal & 8.795 & 474.00 \\
\hline Mínimo operativo (vol. morto) & 5.754 & 465.23 \\
\hline
\end{tabular}

A Figura 2 mostra as trajetórias, $D_{j}, j=1, \ldots 9$, de um histórico de nove anos (1981 a 1989) de vazões diárias, correspondente a 181 dias do período de chuva. Nota-se, neste gráfico, uma dispersão muito maior nos dias mais chuvosos (entre os dias 60 e 80) do que nos dias iniciais e finais do período de cheia. Esses dados foram usados para estimar os coeficientes de drift e difusão da carga $Y(t)$.

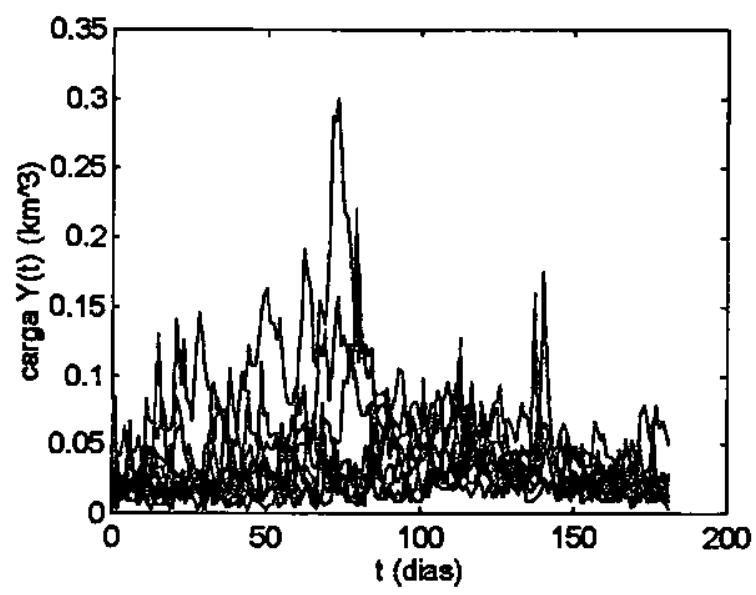

Figura 2. Trajetória de carga, no período de chuva, nos anos de 1981 a 1989

As Figuras 3 e 4 mostram, respectivamente, as estimativas dos coeficientes de drift e difusão obtidas por métodos de máxima verossimilhança e inferência bayesiana. Pode-se notar que o coeficiente de drift coincide nos dois casos, porém o coeficiente de difusão mostra-se menor quando 
estimados pelo método bayesiano. Isso pode ser justificado por serem poucos os anos de dados ( 9 anos) para calcular estimativas de máxima verossimilhança para esse coeficiente.
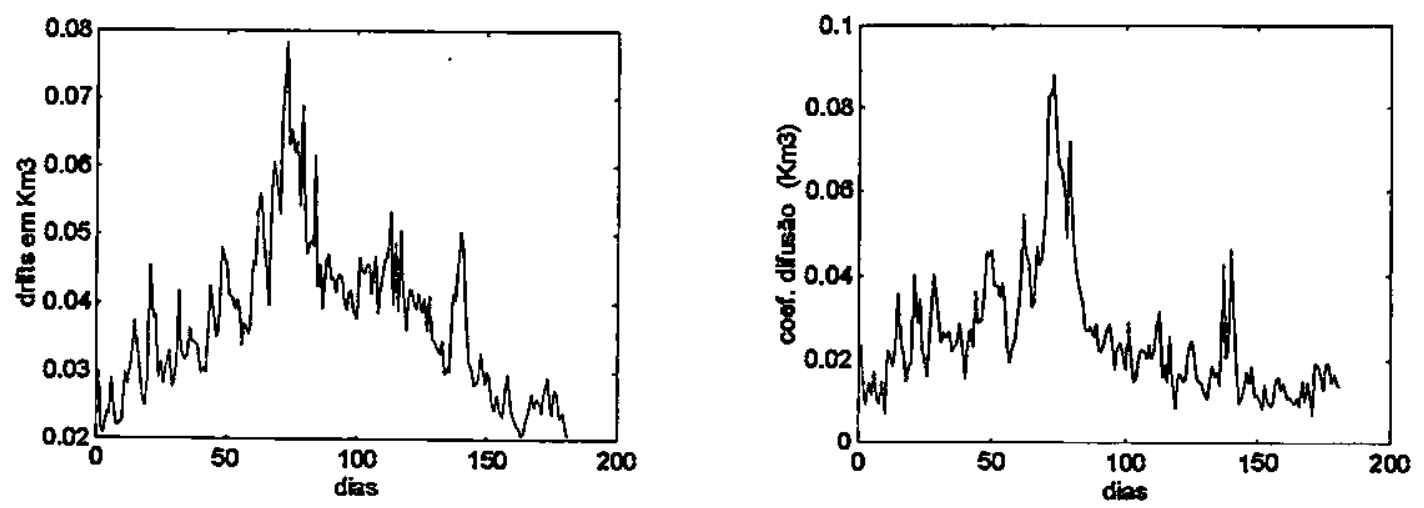

Figura 3. Estimativa de máxima verossimilhança dos coeficientes de drift $\alpha_{i}$ e difusão $\sigma_{i}^{2}$ para $i=1, \cdots 181$, dias
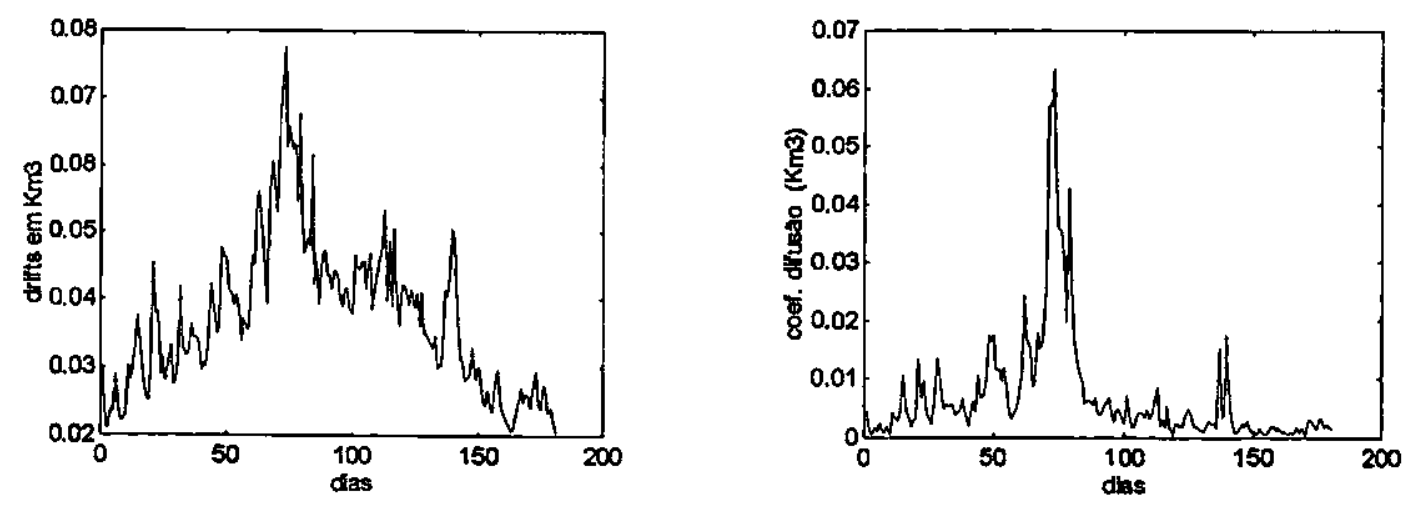

Figura 4. Estimativa bayesiana dos coeficientes de drift $\alpha_{i}$ e difusão $\sigma_{i}^{2}$ para $i=1, \cdots 181$, dias

Um exemplo das densidade a posteriori para os coeficientes de drift e difusão obtidas quando se adota a abordagem bayesiana é apresentada nas Figuras 5(a) e 5(b). Pode-se observar que, para o drift, obteve-se uma distribuição marginal normal e para o coeficiente de difusão, uma distribuição 
marginal gama-inversa para o dia $i=73$ do período chuvoso. Para os outros dias, os gráficos são semelhantes e por isso não serão apresentados.

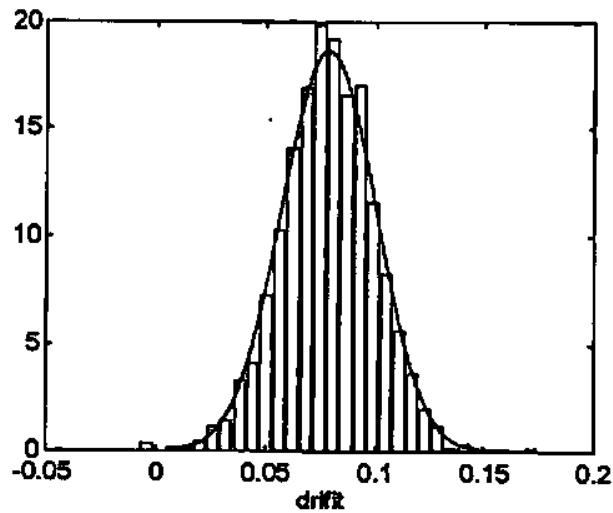

(a)

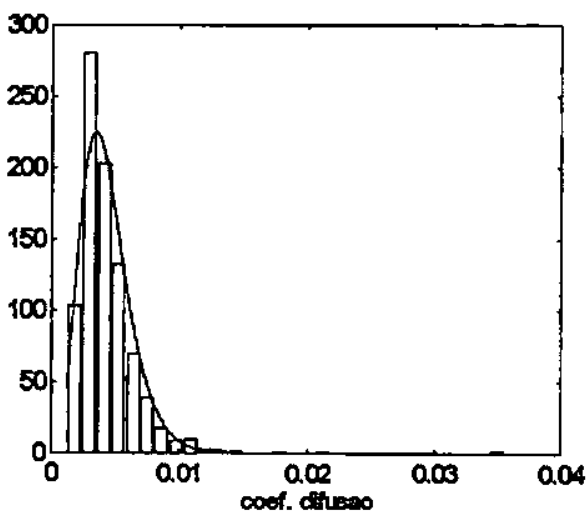

(b)

Figura 5: Exemplo de densidades a posteriori estimadas por MCMC, do coeficiente de drift (a), e de difusão (b) para o dia $i=73$

Para verificar a convergência do método de MCMC, foi utilizado o critério de convergência de Gelman-Rubin para cada uma das estimativas de drift e coeficiente de difusão. O maior valor obtido para o drift foi de 1.0066 e para o coeficiente de difusão foi de 1.0671 . Com isso, as convergências são satisfeita.

Na figura 6, pode-se observar que a convergência que utiliza critério de convergência de Gelman-Rubin das estimativas de drift e coeficiente de difusão para o dia $i=73$ do período chuvoso. Para os outros dias, os gráficos são semelhantes e por isso não serão apresentados. 


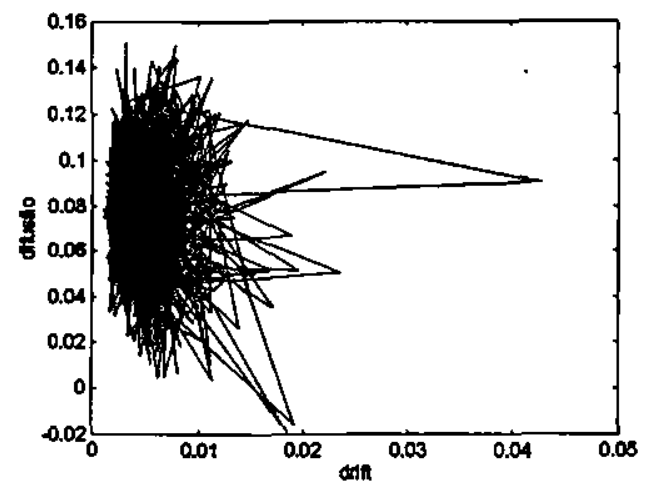

Figura 6: Exemplo do critério de convergência de Gelman-Rubin para as estimadas por MCMC, drift $\times$ difusão para o dia $i=73$

A Figura 7 apresenta uma previsão para um periodo de cheia com 181 dias, que foi feita tomando-se o percetil $95 \%$ das trajetórias mostradas na Figura 2. Essa previsão diária é substituída na equação (5.12) juntamente com os estimadores de máxima verossimilhança para se obter a estimativa de máxima verossimilhança dos volumes de espera para um determinado risco $p$. As estimativas bayesianas desses volumes de espera são obtidas substituindo-se as previsões de cheias diárias na equação (5.18).

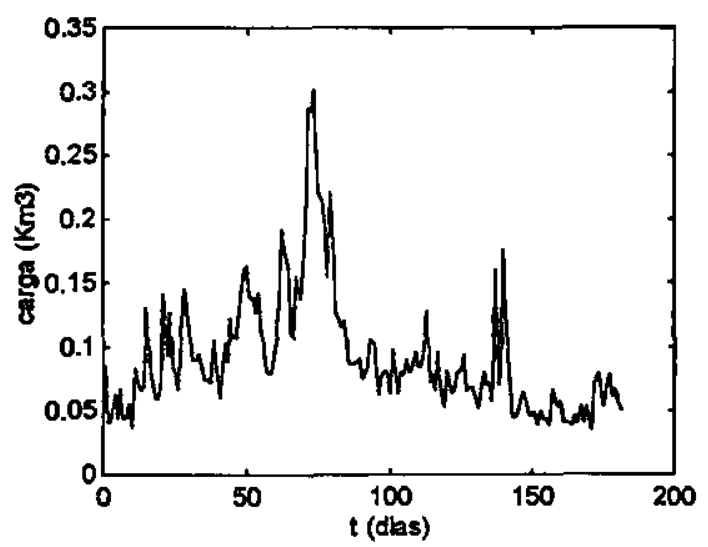

Figura 7. Previsão de cheia (carga) para um periodo de 181 dias. 
As Figuras 8(a) e 8(b) apresentam, respectivamente, os volumes de espera, estimados pelos métodos de máxima verossimilhança e bayesiano, para um risco de falha fixo em $p=5 \%$. Nota-se nesses gráficos que o volume de espera máximo é estimado para o 730 dia do período de chuva. Nota-se que o volume de espera estimado pelo método de máxima verossimilhança é maior do que o estimado por inferência bayesiana.

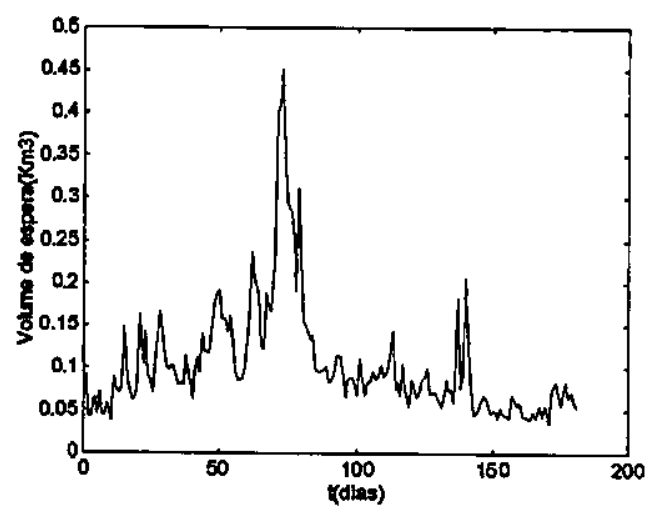

(a)

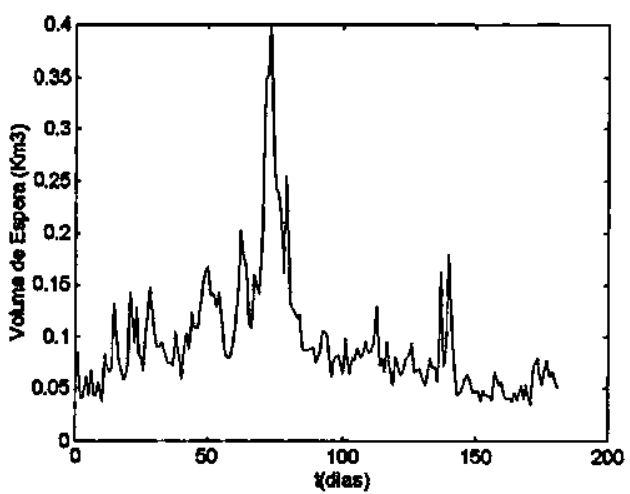

(b)

Figura 8. (a) Volume de espera estimado pelo método de máxima verossimilhança e (b) pelo método bayesiano

0 volume de espera máximo estimado por método de máxima verossimilhança é de 0.4510 $\mathrm{km}^{3}$, enquanto a estimativa bayesiana é de $0.3854 \mathrm{~km}^{3}$. As estimativas desses volumes calculados por outros métodos já consagrados para resolver o problema de controle de cheias, tais como o método da Curva Volume $\times$ Duração (CVD) [Beard-1963] e o método das Trajetória Críticas (TC) [Kelman -1987], são dadas na Tabela 2. 


\subsection{Comparação entre o Método Proposto e os Métodos Existentes}

A comparação do método proposto com os métodos da Curva Volume $\times$ Duração (CVD) $\mathrm{e}$ método das Trajetórias Críticas (TC) é feita aqui analisando-se o resultado da simulação da operação do reservatório para um histórico de vazões diárias, durante o período de 9 anos (1981-1989) de cheias. Cada período contém 181 dias e é referente aos meses de novembro a maio. Apesar desses dados refletirem uma simulação real do reservatório, esse histórico é muito pequeno para fins de simulação e para que as conclusões tiradas aqui sejam consideradas definitivas. No entanto, esses dados nos permitem uma avaliação das principais características entre os métodos. As variáveis analisadas são apresentadas na Tabela 2.

Tabela 2. Comparação do método proposto com outros métodos

\begin{tabular}{|l|l|l|l|l|}
\hline Métodos & Max. Veross. & Bayes. & CVD & TC \\
\hline Vol. $\left(\mathrm{Km}^{3}\right)$ & 0.4510 & 0.3854 & 0.4986 & 0.0647 \\
\hline Num. dias com falha & 9 & 9 & 6 & 19 \\
\hline Uso do vol. útil (\%) & 95.3 & 96.5 & 83.6 & 99.8 \\
\hline Energ. perdia (10 ${ }^{3} \mathrm{MW}$-dia) & 1.052 & 0.811 & 4.188 & 0.011 \\
\hline
\end{tabular}

A primeira variável dessa análise é o máximo volume de espera alocado por cada método. Notase que o volume de espera constante (adotado para os 181 dias do periodo de cheia) determinado pelo método da Curva Volume $\times$ Duração é muito próximo do volume de espera máximo obtido pelo método proposto. Aqui se destaca a primeira vantagem do método proposto, em que os volumes dessa ordem de grandeza são alocados somente por poucos dias do período de cheia (ver Figuras 8(a) e 8(b)). No caso do método das trajetórias críticas, o máximo volume de espera é muito inferior aos demais. No entanto, a validade desse valor é questionada por ele ter sido determinado 
usando-se somente os dados históricos, uma vez que esse método exige a simulação de um grande número de séries geradas.

O número de dias em que o reservatório falha quando cada um dos métodos é adotado é uma variável também usada na comparação. Na Tabela 2, nota-se que o método proposto e o método (CVD), quando aplicados, levam a aproximadamente o mesmo número de dias em que o reservatório falha. Esses dias ocorreram todos no ano de 1989. No caso do método das trajetórias críticas, constatou-se um número muito maior de dias com falha 6 dias distribuídos da seguinte forma: 3 dias no ano de 1982, 1 dia no ano de 1984, 2 dias no ano de 1988 e 13 dias no ano de 1989.

Outra característica a ser observada é que método (CVD) aloca um volume de espera constante para todo período de cheia, enquanto o método proposto e o método da (TC) calculam um volume para cada dia desse período. Essa característica permite um uso melhor da capacidade de armazenamento do reservatório. Este uso é apresentado em termos de percentual utilizado do volume útil. A Tabela 2 mostra que o método (CVD) deixa uma capacidade ociosa no reservatório em média de $16.4 \%$ do volume útil. Já o método proposto usa em média $96.5 \%$ quando a abordagem bayesiana é adotada. Esse resultado afeta diretamente a produção de energia elétrica da usina hidroelétrica de Xavantes como mostram os valores de energia perdida quando cada método é usado, ou seja, o método (CVD) perde em média 4 vezes mais energia que os outros métodos.

Comparando-se as abordagens bayesiana e a de máxima verossimilhança, nota-se, com os dados da Tabela 2, que, apesar de o máximo volume de espera alocado pelo método máxima verossimilhança ser ligeiramente maior que o bayesiano, este se mostra mais vantajoso quanto à economia de energia. Isso se deve certamente ao maior uso do volume útil obtido com o método bayesiano. 


\section{Capítulo 7}

\section{Conclusão e Propostas Futuras}

Neste trabalho foi apresentado o problema de controle de cheias no Brasil no qual se considerou a vazão afluente ao reservatório como sendo uma carga, e a capacidade do reservatório em conter esta cheia como uma resistência. Aqui, carga e resistência foram modelados como processo estocástico do tipo difusão.

Com este método, foi possível fazer uma alocação dinâmica do volume de espera durante o período chuvoso associado a um determinado risco de falha do reservatório. $O$ cálculo do volume de espera deu-se de duas maneiras: a primeira, utilizando-se descarga de referência constante e a segunda, descarga de referência variável.

Foram utilizadas duas técnicas de estimação dos parâmetros dos modelos: o método de máxima verossimilhança e a inferência bayesiana. No segundo, utilizou-se o algoritmo de simulação de Monte Carlo em Cadeia de Markov (MCMC) e construiu-se um estimador de Monte Carlo para fazer alocação do volume de espera associado a um risco de falha de $5 \%$.

Apresentou-se um estudo de caso para o reservatório de Xavantes, no qual se utilizou o modelo com descarga de referência constante, que foi comparado com outros métodos existentes. Para este estudo, utilizou-se um histórico de nove anos (1981-1989) de vazões diárias correspondentes a 181 dias do período chuvoso.

A partir dos resultados obtidos na tabela 2, pode-se observar que o volume máximo obtido pelos métodos de máxima verossimilhança é bem próximo do método da curva volume $\times$ duração. 
Entretanto, o segundo deixa uma capacidade ociosa do reservatório que afeta diretamente a geração de energia elétrica, principalmente no término da estação chuvosa, fazendo que na entrada da estação seca o reservatório não esteja cheio.

O volume máximo obtido pelo método bayesiano foi menor do que o de máxima verossimilhança mostrando-se mais vantajoso pois o volume útil do reservatório foi maior, havendo, assim, um menor desperdício de energia.

Com o uso de descarga de referência constante como politica ótima de operação todos os métodos falharam. Uma explicação para este fato é que ela não leva em conta o volume já alocado no dia anterior e o volume a ser alocado supera o limite permitido, fazendo que o operador tenha que defluir mais que o permitido, o que causa danos a jusante. Uma possível solução para esse problema seria o uso de descarga de referência variável.

O cálculo do volume de espera usando-se descarga de referência variável não foi apresentado no estudo de caso, pois é necessário uma política ótima de operação do reservatório para que esse caso seja resolvido. Além disso o cálculo do risco e do volume de espera só pode ser feito usando-se métodos numéricos para o problema de valor de contorno. Este problema, portanto, é deixado aqui como uma proposta futura. Outra proposta futura é estender o estudo de caso para outros reservatórios do sistema brasileiro. 


\section{Referências Bibliográficas}

ARNOLD, L. Stochastic Differential Equation: Theory and Applications. USA, John Wiley \& Sons, 1974.

BARBOSA, P. S. F. The Choice of River Routing Parameters on Reliability Analysis Applied to Flood Control Purpose. Taipei, Proc. of the sixth IAHR Inter. Symp. On Stoch. Hydraulics, 1992, p. $91-98$.

BEARD, L. R. Flood Control Operation of Reservoir. Jour. of Hydraulic Division, ASCE, Vol. 89, Proc. Paper 3380, p. 8-10, 21-23, 1963.

BOX, G.E.P., TIAO, G.C. Bayesian Inference in Statistical Analysis. Addison-wesley, 1973.

BASAWA, I.V., RAO, B.L.S. Statistical Inference for Stochastic Processes. New York, Academic Press, 1980.

CASELA, G., GEORGE, E.I. Explaining the Gibbs Sampler. The American Statistician, Washigton, v. 46, n. 3, p. 167-174, 1992

DAMÁZIO, Jorge M. Condições de Controlabilidade de Sistemas de Reservatórios para Controle de Cheias e seu Uso na Operação de Sistemas com Multiplos Usos. Rio de Janeiro, 1988. 171 p. Tese (Doutorado) - COPPE, Universidade federal do Rio de Janeiro.

GARABEDIAN, P. R. Partial Differential Equation. New York, John Wiley \& Sons, 1964.

GELMAN, A., CARLIN, J.B., STERN, H.S., RUBIN, D.R. Bayesian Data Analysis. London, Chapman \& Hall, 1995

GELMAN, A., RUBIN, D.R. Inference from Iterative Simulation Using Multiple Sequences. Statistical Sciences, v.7, p. 457-511, 1992.

KELMAN, J. Flood and Hydroeletric Utilization. Rio de Janeiro, $A B R H, R B E$, p. 91-98, 1987.

LOPES, L. A.; DANTAS, H. M. G., DIB, K. R. A Decade of the Brazilian Electrical Sector in the Flood Control. Relatório Técrico, ELETROBRAS, 1986. 
MEON, G. Overtopping Probability of Dams Under Flood Load. Proc. of the Sixth IAHR Inter. On Stoch. Hydraulics, p. 99-106, 1992.

VAL, J. B. R., ANDRADE, M. G. On the Numerical Solution of the Dirichlet Problem For Helmlholtz Equation. Amer. Math. Letter, V. 9, N. 2, p. 85-89, 1996.

YEN, B. C. Stochastic Methods and Reliability Analysis in Water Resources. Computer Methods \& Water Resources, Computational Hydrology, p. 183-197, 1988. 


\section{Apêndice A}

\section{A.1. Método da Curva Volume $\boldsymbol{X}$ Duração}

Este método relaciona, para cada intervalo de tempo com duração de $d$ dias consecutivos, o máximo volume afluente, definido por:

$$
v a(d)=\underset{0<t<h-d+1}{\max }\left(\sum_{j=0}^{d-1} q(t+j) \Delta t\right)
$$

onde: $v a(d) \quad$ - máximo volume afluente para a duração de $d$ dias;

d - duração em dias;

$q(t+j)-$ vazão média no dia $t+j$;

$\Delta t \quad-$ intervalo de discretização $(1$ dia $=864000 \mathrm{~s})$

$h \quad \rightarrow$ número de dias da estação chuvosa

$t \quad-$ dia

Utilizando-se uma série histórica de vazões naturais médias diárias e admitindo-se uma vazão defluente máxima que não cause danos a jusante, pode-se definir, para cada ano hidrológico, o volume vazio necessário para absorver cheias com qualquer duração. Este volume é denominado volume de espera para o ano $i$. A duração associada a este volume é chamada duração crítica. $\mathbf{O}$ volume de espera pode ser representado pela expressão abaixo:

$$
v e(i)=\max [v a(d)-d \times q r \times \Delta t, d=1,2,3, . ., h]
$$


onde: $v e(i)$ - volume de espera para o periodo chuvoso do ano hidrológico i;

gr - descarga de referência.

O objetivo deste método é escolher o volume de espera a ser alocado. No entanto, nada garante que o histórico se repetirá no futuro. A solução mais comum conduz a um estudo de freqüência e à adoção de uma distribuição teórica de probabilidades. São levantadas da série histórica amostras de eventos máximos, de várias durações.

É possivel construir a Curva Volume $\times$ Duração associada a uma probabilidade $p^{\prime}$ fixa de emergência, ajustando-se uma distribuição de probabilidade a cada duração de $d$ dias.

Neste caso, $v a(d)$ é dada por:

$$
P[V A(d)>v a(d)]=p^{\prime}
$$

Uma das dificuldades deste método deve-se à variação amostral, que faz que a curva va(d) não seja côncava, como é esperado.

A questão fundamental que se coloca para a utilização do método é relativa a qual distribuição teórica de probabilidade deve ser ajustada.

Uma outra dificuldade da aplicação do método Curva Volume $X$ Duração é que o mesmo informa somente um único volume de espera para toda a estação chuvosa.

Para maiores informações sobre o método Curva Volume $X$ Duração, veja Beard (1963) e Damázio (1988) 


\section{A.2. Método das Trajetórias Críticas}

Este método permite determinar o volume de espera para cada dia da estação chuvosa com uma probabilidade de emergência $\boldsymbol{p}^{\prime}$, previamente estabelecida.

O método utiliza um algoritmo recursivo das vazões naturais médias diárias. Para cada registro anual identifica-se um periodo chuvoso de $h$ dias. Logo, ao início do último dia, o volume de espera deverá ser igual a:

$$
v e(h . i)=\max [0,(q(h, i)-q r) . \Delta t]
$$

onde: ve - volume de espera;

h - índice do último dia;

$i \quad-$ índice do ano;

$q(h, i)$ - vazão média diária no dia $h$ do ano $i$;

gr - descarga de restrição;

$\Delta t \quad-$ intervalo de discretização $(1 \mathrm{dia}=864000 \mathrm{~s})$

No início do penúltimo dia, o volume de espera deverá ser igual a:

$$
v e(h-1 . i)=\max [0,(q(h-1, i)-q r) . \Delta t+v e(h, i)]
$$

De uma forma geral:

$$
v e(t-1, i)=\max _{t=0,1, \ldots, h}[0,(q(t-1, i)-q r) . \Delta t+v e(t, i)]
$$


A evolução do volume de espera para o $i$-ésimo ano denomina-se trajetória crítica. A mesma define uma fronteira para a operação do reservatório, ou seja, uma curva limite.

A curva limite garantirá que em nenhum instante, não importando o ano sorteado, ocorrerá uma vazão de defluente superior à descarga de restrição, desde que a vazão defluente seja igual a $q r$ quando o volume armazenado no reservatório estiver sobre ou abaixo da mesma.

Considerando-se uma série histórica de $n$ anos, conseqüentemente $n$ trajetórias, a curva limite garantirá a não ocorrência de violação da descarga de restrição, na hipótese de qualquer situação hidrológica observada no passado.

A curva limite é definida por:

$$
v^{\prime}(t)=\max [v e(t, i) ; i=1,2, \ldots, n] ; n=1,2, \ldots, h
$$

A alocação desta curva limite seria inviável, sem a aceitação de alguma probabilidade de emergência $p^{\prime}$. Poderia definir o conjunto de $k$ trajetórias críticas $C p^{\prime}$ não protegidas pelo volume de espera a ser alocado. Como o objetivo é a geração de energia tal conjunto deve minimizar a alocação de volume de espera, definido pela seguinte função:

$$
B\left[V p^{\prime}(1), V p^{\prime}(2), \ldots, V p^{\prime}(h)\right]
$$

onde: $V p^{\prime}(i)$ - volume de espera alocado no dia $i$ para uma probabilidade $p^{\prime}$.

Para minimizar, $B$ podem-se utilizar dois critérios:

(i) Obter o conjunto $C p^{\prime}$ que implique uma envoltória que minimize o volume de espera médio; 
(ii) Formar o conjunto $C p^{\prime}$ de trajetórias críticas que, considerando-se um intervalo, apresente os volumes de espera máximos.

Em ambos os critérios, é necessário determinar o volume de espera dia a dia para uma probabilidade de emergência $p^{\prime}$, previamente estabelecida.

Para maiores detalhes, veja Kelman (1987) e Damázio (1988) 


\section{Apêndice B}

\section{B.1 Abordagem Bayesiana e Simulação de Monte Carlo em Cadeia de Markov}

Com base no teorema da Bayes, pode-se considerar um problema genérico no qual se tem um conjunto de dados, para fazer inferência sobre um parâmetro.

Seja $y=\left(y_{1}, \ldots, y_{n}\right)$ vetor aleatório de $n$ observações. A distribuição de probabilidade de $y$ é dada por $p(y \mid \theta)$, a qual depende do valor do parâmetro $\theta$. Vamos supor ainda que $\theta$ tem uma distribuição de probabilidade dada por $p(\theta)$.Então:

$$
p(y \mid \theta) \cdot p(\theta)=p(y, \theta)=p(\theta \mid y) p(y)
$$

Dado y observado, a distribuição condicional de $\theta$ é dada por:

$$
p(\theta \mid y)=\frac{p(y \mid \theta) p(\theta)}{p(y)}
$$

onde $p(y)= \begin{cases}\int_{\theta \in \Theta} & p(y \mid \theta) p(\theta), \theta \text { contínuo } \\ \sum_{\theta \in \Theta} p(y \mid \theta) p(\theta), \theta \text { discreto }\end{cases}$ sendo $\Theta$ o conjunto correspondente ao domínio do parâmetro $\theta$.

A expressão $(\mathrm{B}, 1)$ é geralmente referida como Teorema de Bayes. Nessa expressão cada componente possui uma interpretação específica em Inferência Bayesiana. 


$$
\text { - } p(\theta) \text { - Priori }
$$

Em inferência bayesiana, o parâmetro de interesse geralmente denotado por $\theta$ é considerado uma variável aleatória com uma função de probabilidade desconhecida, chamada de distribuição a priori.

A distribuição a priori expressa todo o conhecimento ou ignorância (incerteza) que o pesquisador possui a respeito do parâmetro, antes da realização do experimento ou antes da observação dos dados que envolvem tal parâmetro.

\section{- $p(y \mid \theta)$ - Verossimilhança}

A função de verossimilhança é considerada como uma função do parâmetro de interesse para um conjunto de dados observados. Logo, $p(y \mid \theta)$ em (1) pode ser considerada como uma função $\theta$ mas não de $y$.

$$
\text { - } \boldsymbol{p}(\theta \mid y) \text { - Posteriori }
$$

A distribuição a posteriori $p(\theta \mid y)$ é uma função de probabilidade, que informa o que é conhecido a respeito de $\theta$ depois da realização do experimento, através da função de verossimilhança combinada com a informação a priori.

$$
\text { - } p(y) \text { - Constante de Normalização }
$$

$A$ constante de normalização garante que a distribuição a posteriori $p(\theta \mid y)$ integre ou some um.

Pode-se escrever o teorema de Bayes como:

$$
p(\theta \mid y) \propto p(y \mid \theta) p(\theta)
$$


isto é, a distribuição a posteriori de $\theta$, dado $y$, é proporcional ao produto da distribuição a posteriori e da função de verossimilhança de $\theta$, considerados os dados.

Para uma melhor compreensão sobre a teoria de bayesiana, sugere-se uma consulta a: Box (1973) e Gelmam (1995)

\section{B.2. 0 amostrador de Gibbs}

O amostrador de Gibbs é uma técnica de simulação de observações de uma distribuição, sem que se tenha de calcular analiticamente a densidade. É essencialmente um esquema iterativo de amostragem de uma cadeia de Markov, cujo núcleo de transição (ou função geradora) é formado pelas densidades condicionais completas.

Apesar de o amoṣtrador de Gibbs ser perfeitamente aplicável num contexto genérico de uma distribuição qualquer, vai-se descrevê-lo sob o enfoque bayesiano, isto é, como ferramenta para a construção de amostras de uma distribuição a posteriori.

Seja $\theta=\left(\theta_{1}, \theta_{2}, \ldots, \theta_{k}\right)$ um vetor paramétrico $k$-dimensional, onde $\theta_{i}, i=1, \ldots, k$, são variáveis aleatórias cuja densidade (posteriori) conjunta é dada por:

$$
p(\theta)=p\left(\theta_{1}, \ldots, \theta_{k} \mid \mathfrak{D}\right)
$$

onde $\mathfrak{D}$ denota o conjunto de dados observados.

Supondo o interesse em obter caracteristicas das densidades marginais $p\left(\theta_{i}\right), i=1, \ldots, k$, mas o cálculo analítico ou numérico é extremamente complicado. Neste caso, o amostrador de Gibbs fornece um método alternativo para a obtenção das marginais. A técnica de simulação permite gerar 
uma amostra $\theta_{i}^{(1)}, \theta_{i}^{(2)}, \ldots, \theta_{i}^{(m)} \sim p\left(\theta_{i}\right)$, sem o conhecimento de $p\left(\theta_{i}\right), i=1, \ldots, k$. Para um número amostral suficientemente grande, a média, a variância e outras características de $p\left(\theta_{i}\right)$ podem ser calculadas.

Partindo-se do pressuposto de que as densidades a posteriori condicionais $p\left(\theta_{i} \mid \theta_{(i)}\right)$, onde $\theta_{(i)}=\left(\theta_{1}, \ldots, \theta_{i-1}, \theta_{i+1}, \ldots, \theta_{k}\right)$ são disponíveis sob uma forma padronizada, o algoritmo do amostrador de Gibbs é descrito da seguinte forma:

(i) admitam-se valores iniciais arbitrariamente $\theta^{(0)}=\left(\theta_{1}^{(0)}, \theta_{2}^{(0)}, \ldots, \theta_{k}^{(0)}\right)$ e inicialize-se o contador de interações da cadeia em $j=1$;

(ii) obtenha-se um novo valor $\theta^{(j)}=\left(\theta_{1}^{(j)}, \theta_{2}^{(j)}, \ldots, \theta_{k}^{(j)}\right)$ a partir de $\theta^{(j-1)}$ através de sucessivas gerações de valores:

$$
\begin{gathered}
\theta_{1}^{(j)} \sim p\left(\theta_{1} \mid \theta_{2}^{(j-1)}, \ldots, \theta_{k}^{(j-1)}\right) \\
\theta_{2}^{(j)} \sim p\left(\theta_{2} \mid \theta_{1}^{(j)}, \theta_{3}^{(j-1)}, \ldots, \theta_{k}^{(j-1)}\right) \\
\cdot \\
\cdot \\
\cdot \\
\theta_{k}^{(j)} \sim p\left(\theta_{k} \mid \theta_{1}^{(j)}, \ldots, \theta_{k-1}^{(j)}\right)
\end{gathered}
$$

(iii) mude-se o contador $j$ para $j+1$ e faça o retorno a (ii) até obter a convergência.

Repetindo o processo $n$ vezes, obtemos $\left(\theta_{1 s}^{(j)}, \theta_{2 s}^{(j)}, \ldots, \theta_{k s}^{(j)}\right), s=1,2, \ldots, n$. Admitindo convergência, qualquer característica da densidade marginal $p\left(\theta_{i}\right)$ pode ser obtida.

Para maiores detalhes, veja-se Casella-George (1992) 


\section{B.3. O critério de convergência de Gelman e Rubin}

Uma forma simples de monitorar a convergência é utilizar $m \geq 2$ cadeias de Markov independentes, em paralelo. Os pontos iniciais devem ser sobredispersos em relação à distribuição procurada. Após a convergência, todas as cadeias devem ter o mesmo comportamento e, supondo que isso aconteça na $t$-ésima iteração, usam-se os valores $\theta_{j}, \theta_{j+h}, \theta_{j+2 h}, \ldots, \theta_{j+n h}$ onde $j \geq t$, como uma amostra aleatória da distribuição desejada. Para que essa amostra seja i.i.d., $h$, tem que ser um número suficientemente grande, tal que permita haver independência entre as realizações.

Gelman e Rubin (1992) propбem um método de verificação de convergência, baseado em técnicas de análise de variância, onde a convergência é aceita quando a variância entre as cadeias for bem menor do que a variância dentro de cada cadeia. Se cada seqüência possui tamanho $2 n$, descartam-se as primeiras $n$ iterações e utilizam-se as últimas $n$.

Para cada parâmetro de interesse, calcula-se:

- a variância entre as cadeias: $E=\frac{n}{m-1} \sum_{i=1}^{m}\left(\overline{\theta_{i}}-\bar{\theta}\right)^{2}$;

- a variância dentro das cadeias: $D=\frac{1}{m(n-1)} \sum_{i=1}^{m} \sum_{j=1}^{n}\left(\theta_{i}^{(j)}-\overline{\theta_{i}}\right)^{2}=\frac{1}{m} \sum_{i=1}^{m} S_{i}^{2}$

onde $\bar{\theta}_{i}$ é a média das observações da cadeia $i$ e $\bar{\theta}$ é a média dessas médias, $i=1, \ldots, m$. Então, a variância da distribuição desejada pode ser estimada por $\hat{\sigma}^{2}=\frac{n-1}{n} D+\frac{1}{n} E$ e a média por $\hat{\mu}=\bar{\theta}$. Pode-se melhorar na estimativa mais precisa da distribuição desejada, admitindo-se para a variabilidade amostral das estimativas, $\hat{\mu}$ e $\hat{\sigma}^{2}$. $O$ resultado é uma distribuição t'Student aproximada para $\theta$ com centro $\hat{\mu}$, escala $\sqrt{\widehat{V}}=\sqrt{\widehat{\sigma}+E / m n}$ e graus de liberdade $d f=2 \widehat{V} / V \widehat{a} r(\widehat{V})$, onde

$$
\operatorname{Var}(\widehat{V})=\left(\frac{n-1}{n}\right)^{2} \frac{1}{m} \operatorname{Var}\left(S_{i}^{2}\right)+\left(\frac{m+1}{m n}\right) \frac{2}{m-1} E+
$$




$$
+2 \frac{(m+1)(n-1)}{m n^{2}} \frac{m}{n}\left[\operatorname{cov} v\left(S_{i}^{2}, \bar{\theta}_{i}^{2}\right)-2 \bar{\theta} \hat{c o v}\left(S_{i}^{2}, \bar{\theta}_{i}\right)\right]
$$

e onde as variâncias e covariâncias estimadas são obtidas dos $m$ valores amostrais de $\bar{\theta}_{i}$ e $S_{i}^{2}$; $d f \rightarrow \infty$ quando $n \rightarrow \infty$.

Deve-se monitorar a convergência da simulação iterativa, calculando-se o fator pelo qual a escala da distribuição atual para $\theta$ deve ser reduzida, se as simulações forem contínuas no limite $n \rightarrow \infty$. A redução potencial de escala é, portanto, dada por $\sqrt{\widehat{R}}=\sqrt{(\widehat{V} / \widehat{D}) d f /(d f-2)}$, que decresce para 1 quando $n \rightarrow \infty$. Se essa redução é um valor alto, deve-se aumentar o número de iterações sobre a distribuição desejada. Desde que $\hat{\mathrm{R}}$ seja próximo de 1 para todas as estimativas escalares de interesse, a convergência é admitida e as amostras (cadeias) finais das iterações selecionadas são ïd.

Maiores detalhes sobre critério de convergência são fornecidos por Gelman-Rubin (1992). 